\title{
Neurexin and Neuroligins Jointly Regulate Synaptic Degeneration at the Drosophila Neuromuscular Junction based on TEM Studies
}

\section{Guangming Gan ( $\nabla$ ganguangm@seu.edu.cn )}

Southeast University

\section{Zhang Chenchen}

Southeast University

\section{Gao Xiang}

Southeast University

Lu Keze

Southeast University

\section{Xie Wei}

Southeasty University

\section{Geng Junhua}

Southeast University

\section{Research article}

Keywords: Drosophila, Neuromuscular junction, Synapticdegeneration, Neurexin, Neuroligins, Transmission electron microscopy

Posted Date: July 2nd, 2020

DOl: https://doi.org/10.21203/rs.3.rs-36148/v1

License: (c) (1) This work is licensed under a Creative Commons Attribution 4.0 International License. Read Full License 


\section{Abstract}

Background: The Drosophila larval neuromuscular junction (NMJ) is a well-known model system and is often used to study synapse development and signal transmission in neurology. Here, we show the synaptic degeneration at NMJ boutons, primarily based on transmission electron microscopy (TEM) studies.

Methods: To this aim, we extensively acquire the ultrastructure of diffuse NMJ boutons using continuous sectioning and transmission electron microscope in the wide type and the dneurexin (dnrx) and dneuroligins (dngs) mutant.

Results: When degeneration starts, the subsynaptic reticulum (SSR) swells, retracts and folds inward, and then, the residual SSR degenerates into a disordered, thin or linear membrane with a shrinking postsynaptic area (PSA) and axon terminal. The axon terminal begins to degenerate from the central region, and the T-bar structure detaches from the presynaptic membrane with clustered synaptic vesicles to accelerate large-scale degeneration, accompanied by retraction of synaptic vesicles and mitochondria. There are two degeneration modes for clear synaptic vesicles. In the first mode, synaptic vesicles without actin filaments degenerate on the membrane with ultrafine spots and collapse and disperse to form an irregular profile with dark ultrafine particles. In the second mode, clear synaptic vesicles with actin filaments degenerate into dense synaptic vesicles, form irregular dark clumps without a membrane, and collapse and disperse to form an irregular profile with dark ultrafine particles. Last, all residual membranes in NMJ boutons, including presynaptic and postsynaptic membranes, become very thin, most of the SSR degenerates into a linear shape, and all the residual elements in axon terminals, such as synaptic vesicles, mitochondria, the cytoskeleton, and the T-bar, degenerate in situ and eventually form a cluster of dark ultrafine particles. Axon terminals and elements within axon terminals degenerate with the postsynaptic SSR, and swelling and retraction of the SSR occurs prior to axon terminal degradation, which degenerates faster and with more intensity than the SSR. NMJ bouton degeneration occurs under normal physiological conditions, but degeneration in $d n r x$ and $d n l g s$ mutant is accelerated. Furthermore, there is a synergistic effect in $d n r x, d n l g s$ double mutants and $d n l g 2 ; d n l g 3$ double mutants that promotes degeneration of NMJ boutons.

Conclusions: NMJ bouton degeneration occurs under normal physiological conditions but is accelerated in $d n r x$ and $d n l g s$ mutants. Furthermore, there is a synergistic effect in $d n r x$,dnlgs double mutants and $d n \lg 2$; dnlg3 double mutants that promotes degeneration of NMJ boutons, which suggests that both neurexin and neuroligins play a vital role in preventing synaptic degeneration. This study proposes the model of NMJ bouton degeneration patterns, which is very conducive to in-depth study of neurodegeneration.

\section{Background}


Efficient signal communication is carried out by means of a massive number of delicate synapses among neurons. During development of the nervous system, neurons tend to produce redundant synaptic connections that will be pruned $[1,2]$ or degenerated $[3-6]$ and then eliminated $[7,8]$ by astrocytes $[9-11]$, microglia [12,13], and Schwann cells [14-17]. Therefore, the stability of synaptic boutons is a dynamic balance among growth [12], pruning, degeneration, and elimination processes. Synapse degeneration is a complicated process that includes retraction $[18,19]$ and degradation $[14,20]$ of presynaptic and postsynaptic components, such as synaptic vesicles [13, 15, 19, 21-23], microtubules [2], and postsynaptic PSD [24]. Disordered elimination after synaptic degeneration can lead to autism [25] and other neurological diseases.

Loss of neurons in the brain and spinal cord leads to neurodegenerative diseases, such as Alzheimer's disease, Parkinson's disease, and amyotrophic lateral sclerosis. In the peripheral nervous system, degeneration of NMJs occurs prior to cell soma degeneration [26, 27]. Transmission electron microscopy is a powerful tool for studying synaptic degeneration due to its ultrahigh resolution. In degenerating terminals, the synaptic vesicles will be reduced and collapse, with shrinking of the synaptic terminal [13, 19]. However, current studies of synaptic bouton degeneration primarily focus on degradation of synapses and mitochondria, cytoskeletal disorders, and reduction of synaptic vesicles, while few studies have reported on the collapse and degradation of synaptic vesicles. The morphological collapse and degradation of synaptic vesicles is rarely reported in the literature and can technically be investigated only using electron microscopy.

The Drosophila larval neuromuscular junction (NMJ) is a well-known model system for studying synaptic development, signal transmission and neurological disease. Synapse retraction often precedes synapse degeneration in NMJ boutons in Drosophila. Synaptic debris and synaptic footprints are remnants of presynaptic boutons that retract from normal NMJ boutons in Drosophila. Synaptic debris is small in size and lacks synapsin as a marker of the clear synaptic vesicles and postsynaptic Dlg protein as a marker of NMJ bouton SSR but has obvious Hrp signals $[28,29]$. Synaptic footprints with SSR membranes and retracted axon terminals have postsynaptic Dlg proteins [30,31] but are almost completely lacking in synapsin and Hrp as markers of the presynaptic membrane [30]. Moreover, the key molecules for synaptic assembly of presynaptic components, such as the microtubule skeleton proteins Futsch [32, 33] and brp [33], are decreased in the early stage of synapse degeneration in neurodegenerative models.

Neurexins $[34,35]$ and neuroligins $[36,37]$ are synaptic cell-adhesion molecules that bridge the synaptic cleft, organize molecules for synapses, mediate trans-synaptic signaling, and shape neural network properties. All of them show strong expression in NMJ pre-and/or postsynaptic compartments, and loss of $d n r x$ and/or $d n l g s$ leads to significant defects in synaptic growth, ranging from abnormal bouton size/bouton number and abnormal active zones to misformed pre- and postsynaptic structures at the Drosophila NMJ. However, most studies on $d n r x$ and $d n / g s$ focus on synapse growth and formation, but the roles of $d n r x$ and $d n l g s$ in synaptic degeneration are poorly understood. 
Therefore, we characterized the ultrastructure of synaptic degeneration at Drosophila larval NMJs, including synaptic vesicles, in the $w^{1118}, d n r x$ and $d n l g s$ lines, as well as in pupae, and the results showed that NMJ bouton degeneration occurs in wild-type Drosophila and is accelerated in dnrx and dnlgs mutants.

\section{Methods}

\section{Animals}

The $w^{1118}$ strain was used as the wild-type control in this study. The following fly mutants were used:

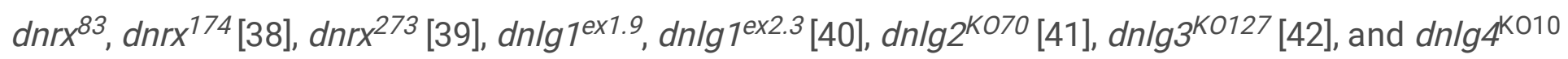
[43]. The double mutants $d n r x^{83} ; d n \lg 3^{K O 127}$ and $d n \lg 2^{K O 70} ; d n \lg 3^{K O 127}$ were generated in our laboratory. All stocks were cultured in standard medium at $25^{\circ} \mathrm{C}$.

\section{TEM analysis of larval NMJ boutons}

TEM was performed according to the procedure described in our previous paper. In brief, wandering thirdinstar larvae were dissected in ice-cold discs in Jan solution $(128 \mathrm{mM} \mathrm{NaCl}, 2 \mathrm{mM} \mathrm{KCl}, 4 \mathrm{mM} \mathrm{MgCl} 2,35$ $\mathrm{mM}$ sucrose, $5 \mathrm{mM}$ HEPES, $\mathrm{pH}$ 7.4) using standard techniques and then fixed with a mixed fixative containing $2 \%$ glutaraldehyde and $2 \%$ formaldehyde (dissolved in $0.1 \mathrm{M}$ sodium cacodylate buffer, $\mathrm{pH}$ 7.4) at $4^{\circ} \mathrm{C}$ overnight. The samples were rinsed with cacodylate buffer several times at $4^{\circ} \mathrm{C}$, postfixed for 2 hours with $1 \% \mathrm{OsO}_{4}$ in $0.1 \mathrm{M}$ cacodylate buffer, and rinsed twice with distilled water. Then, the samples were stained for 2 hours with $2 \%$ saturated uranyl acetate and rinsed twice with distilled water. The specimens were dehydrated in an increasing ethanol series (30\%, 50\%, 70\%, 85\%, 95\%, 100\% twice), passed through propylene oxide twice, and embedded into a sheet in Epon812 (SPI Science). The sheet was serially sectioned with a total of 80 microns using a diamond knife on a Leica UC7 ultrathin microtome at the 6th/7th muscles of the $A_{3}$ or $A_{2}$ segment in one animal; each ultrathin slice was $90 \mathrm{~nm}$ thick. Approximately 30-40 slices were gathered into a group and attached to a grid, and approximately 30 grids were used in each sample. The grids were stained again with $2 \%$ saturated uranyl acetate in 50\% ethanol and then stained with $1 \%$ lead citrate $(\mathrm{pH} 12)$. Last, each ultrathin slice was examined under a transmission electron microscope (Hitachi $\mathrm{H}-7650$ ). More than 20 wild-type animals were analyzed, and 3 animals were analyzed for each of the other strains.

\section{TEM of pupal NMJ boutons}

Pupae (13 hours after pupa) were fixed to dissecting dishes with needles at both ends and covered with a few drops of fixative ( $2 \%$ glutaraldehyde and $2 \%$ formaldehyde in $0.1 \mathrm{M}$ sodium cacodylate buffer, $\mathrm{pH}$ 7.4). Then, the dorsal midline was cut longitudinally with scissors, without removing the internal organs. Forty minutes later, both ends of each pupa were cut to promote fixation, and the pupal samples were fixed for 24 hours in fixative at $4^{\circ} \mathrm{C}$. Then, the pupae were postfixed for 2 hours with $1 \% \mathrm{OsO}_{4}$, stained for 2 hours with $2 \%$ saturated uranyl acetate, dehydrated in an ethanol series, passed through propylene 
oxide, treated with propylene oxide and epoxy resin, embedded and polymerized. Semithin slices along the side of the pupae were prepared and stained with toluidine blue to position type I boutons in NMJs, and thin sections of approximately $90 \mathrm{~nm}$ were prepared, collected and attached to grids. The grids were poststained with $2 \%$ saturated uranyl acetate and $1 \%$ lead citrate $(\mathrm{pH} 12)$ and observed under a transmission electron microscope (Hitachi H-7650).

\section{Preembedding immunogold electron microscopy procedure}

Pre-embedding immunogold electron microscopy was performed as follows [44]. In brief, third-instar larvae were dissected in ice-cold discs in Jan solution using standard techniques and then fixed (4\% formaldehyde, $0.5 \%$ glutaraldehyde, and $10 \%$ saturated picric acid in $0.1 \mathrm{M}$ sodium cacodylate buffer, $\mathrm{pH}$ 7.4) for 4 hours at $4^{\circ} \mathrm{C}$ (the following procedures were carried out at $4^{\circ} \mathrm{C}$ ). The specimens were washed 4 times with $0.1 \mathrm{M}$ sodium cacodylate buffer and perforated with $1 \%$ saponin for 1 hour. Then, specimens were preincubated in $0.5 \%$ BSA (bovine serum albumin) and $0.1 \%$ gelatin with $0.1 \%$ saponin for 1 hour and incubated with a mouse primary antibody (anti-synaptotagmin, 3H2 2D7, 1:10 DSHB; and antisynapsin, 3C11, 1:10; DSHB) for 24 hours. After 4 rinses with $0.1 \%$ Tween-20 in $0.1 \mathrm{M}$ PBS, the samples were preincubated with $0.5 \%$ BSA, $0.1 \%$ gelatin and $0.1 \%$ saponin again for 1 hour; incubated with a 1.4 nm ultrasmall gold-conjugated secondary antibody (goat anti-mouse IgG secondary antibody, Nanoprobes, \#2001, 1:50) for 12 hours; and rinsed 4 times with $0.1 \%$ Tween-20 in $0.1 \mathrm{M}$ PBS. The samples were then postfixed in $2.0 \%$ glutaraldehyde in PBS for 30 min and rinsed several times with distilled water. Silver enhancement (HQS kit; Nanoprobes, \#2012) was performed in a dark room for 25 min, followed by rinsing with distilled water. After rinsing with PBS for $10 \mathrm{~min}$, the samples were osmicated $\left(0.5 \% \mathrm{OsO}_{4}\right)$ in $0.1 \mathrm{M}$ sodium cacodylate for 0.5 hours. All samples were washed three times with distilled water and then stained with $2 \%$ aqueous uranyl acetate for 2 hours. Subsequent gradual dehydration, epoxy resin embedding, trimming and thin sectioning were performed as described above for the NMJ boutons at the 6 th/7th muscles in the $A_{3}$ or $A_{2}$ segment.

\section{Immunochemistry}

Immunostaining of the larval samples was performed as described previously ([44]). Briefly, the wandering larval samples were dissected in Jan solution; fixed in paraformaldehyde at room temperature; washed with PBS and 0.3\% PBST (0.3\% Triton X-100 in PBS); blocked in 1\% BSA for 1 hour; incubated with anti-Hrp (Jackson ImmunoResearch, West Grove, PA), anti- synaptotagmin (1:50), and anti-synapsin (1:50) antibodies at $4^{\circ} \mathrm{C}$ for 2 hours; and incubated with fluorophore-conjugated secondary antibodies (Invitrogen, 1:500) for 1 hour at room temperature. The samples were washed extensively with PBST and mounted in VectaShield mounting medium (Vector Laboratories). Images were collected using an Olympus FV3000 confocal microscope. During observation of degenerated NMJ boutons, the large pinhole of the confocal microscope was adjusted to increase the thickness of a single optical section, and the 3D analysis function of the confocal microscope was utilized to observe the complete NMJ bouton in the 6 th/7th muscles in the $A_{3}$ or $A_{2}$ segment. 


\section{Statistical analysis}

The degenerate boutons in the 6th/7th muscles of A2-A3 segments from Drosophila larval were counted. For each strain, at least 4 segments were counted for confocal microscopy data. The data were analyzed with GraphPad Prism 7 using unpaired, two-tailed $t$ tests.

\section{Results}

\section{The ultrastructure of severely degenerated NMJ boutons in wild-type Drosophila}

In our early research, we analyzed a large number of type I NMJ boutons in wild-type Drosophila flies using TEM [44]. Only type I NMJ boutons between the 6th/7th muscles were presented [45] [46], and they were divided into type lb and type Is. In TEM, the type lb bouton (large) (Fig. 1A) was globular, the regular dense SSR membrane circled around the axon terminal, and clear synaptic vesicles gathered in a T-bar (Fig. 1A-A') before the presynaptic membrane with an obvious synaptic cleft. Type Is boutons are smaller, and the SSR membrane is thinner (Fig. 1B) $[45,46]$. In addition, there was a compact postsynaptic area (PSA) that matched after the postsynaptic membrane in both type I boutons (Fig. 1C-C') [47] and type Ib boutons. In the terminal of type I boutons, organelles, such as mitochondria (Fig. 1A, B), were also present.

However, through analysis of more than 20 flies, we found that degenerated NMJ boutons were rarely observed in wild-type Drosophila. Severely degenerated NMJ boutons showed extremely degenerated axon terminals and extremely severe retraction of the SSR. The degenerated terminals gathered in a small area of the outer muscle (Fig. 1D) with rare retracted SSR membranes (Fig. 1D and D"'), and they were basically vacuolated (Fig. 1D) or contained beaded, overlapping, dark synaptic vesicles (Fig. 1D'-D") and residual synapses (Fig. 1G-G") without mitochondria or T-bars. Compared to presynaptic membranes and postsynaptic membranes in normal synapses with a typical T-bar structure (Fig. $1 A^{\prime}$ and $C^{\prime}$ ), the residual synapse had a significantly thin presynaptic membrane and postsynaptic membrane that were stuck to each other, with almost no synaptic cleft (Fig. 1G-G"). The residual presynaptic membranes and postsynaptic membranes were thin and dark without synaptic clefts or T-bars (Fig. 1E", G-G") and sometimes had a tendency to separate from each other (Fig. 1G-G"), which showed the characteristics of complete synapse degeneration.

The degenerated NMJ boutons were also present inside muscle (Fig. 1E-E"'); some were completely vacuolated (Fig. 1E), but some vacuolated boutons contained degenerated ultrafine particles and had a sparse profile of similar dark synaptic vesicles (Fig. 1E"). Furthermore, larger degenerated NMJ boutons in the outer muscle were filled with agglomerated degenerated products (Fig. 1F) and did not contain clear and dark synaptic vesicles but dark ultrafine particles less than $3 \mathrm{~nm}$ in diameter (Fig. 1F'-F"). The SSR was extremely retracted in all degenerated NMJ boutons (Fig. 1D"', E'", F"'), and residual SSRs were rare. There was no T-bar structure or mitochondria in any of the degenerated NMJ boutons. 
In severely degenerated NMJ boutons in wild-type Drosophila, the axonal terminal retracted and shrank, with retraction and degradation of presynaptic components, such as synaptic vesicles, mitochondria and the T-bar structure, and most SSR membranes were retracted from the NMJ boutons. It is worth noting that normal NMJs were singular and isolated by an SSR (Fig. 1A and B), while the severely degenerated NMJs were close together without an obvious SSR (Fig. 1D, F, F).

\section{Gradual degeneration process of NMJ boutons in wild-type Drosophila}

We observed and confirmed severely degenerated NMJ boutons with full retraction into small boutons and then identified the process of degeneration in wild-type Drosophila (Fig. 2A-C).

In the milder degeneration state (Fig. 2A-A"'), the boutons looked similar to normal globular type lb boutons, but in the center region of the terminal, several dark synaptic vesicles and dark ultrafine particles (Fig. 2A-A') appeared with more clear synaptic vesicles (Fig. 2A') and a normal T-bar (Fig. 2A"). Furthermore, the SSR membrane swelled and withdrew (Fig. 2A'). In a moderately degenerating bouton (Fig. 2B-B"'), the dark synaptic vesicles and dark ultrafine particles increased (Fig. 2B'), and clear synaptic vesicles were present around the normal T-bar (Fig. 2B"). However, the SSR membrane further loosened and withdrew, and some swollen SSR membranes were folded inward (Fig. 2B"'). Then, two severely degenerated type $\mathrm{lb}$ boutons were observed, which were close to each other without a T-bar (Fig. 2C). The two boutons had irregular terminals (Fig. 2C-C"') in which there were many dark ultrafine particles (Fig. $\left.2 C^{\prime \prime}\right)$, few dark synaptic vesicles (Fig. 2C') and few clear synaptic vesicles (Fig. 2C'). The SSR became disordered and collapsed, and the SSR membrane was loose and thin or even absent from some regions of severely degenerated boutons with extremely sparse residual SSR membrane (Fig. $2 \mathrm{C}^{\prime \prime}$ ). It seemed that dark synaptic vesicles were the intermediates, and the dark ultrafine particles were the final product during the collapse and degeneration of clear synaptic vesicles. It is likely that severely degenerated boutons are different from synaptic footprints that contain a relatively complete SSR in TEM and postsynaptic Dlg protein but no Synapsin or Hrp [30,31]. It is worth noting that the deformed axon terminal was not detached from the SSR of degenerated boutons but instead degenerated in situ.

Degeneration of NMJ boutons originated from SSR abnormalities in the wild-type fly. The SSR membrane, synaptic vesicles, and T-bar showed marked degeneration in boutons (Fig. 1-2), but which component is the first to become abnormal remained unknown. We observed that the SSR membrane became loose and swollen in type Ib boutons (Fig. 3A-A") and in type Is boutons (Fig. 3B-B"), but the synaptic vesicles and T-bars were very typical in both types of boutons (Fig. $3 \mathrm{~A}^{\prime \prime}, \mathrm{B}$ ), and the center region of the boutons did not exhibit the degeneration that is shown in Fig. 2A-C. Moreover, there were no dark synaptic vesicles or dark ultrafine particles in type lb boutons or type Is boutons (Fig. 3A-B). NMJ boutons undergo marked degeneration during the process of development in the early pupal stage (6 hours pupa) [48]. We found that the T-bars were typical and that most synaptic vesicles were clear and normal in both type Ib boutons (Fig. 3C, C") and type Is boutons (Fig. 3D, D"), but the SSR membrane was obviously swollen, thin, loose and disordered (Fig. 3C-C', D-D') in the pupal stage (13 hours pupa). Furthermore, there 
were no dark synaptic vesicles or dark ultrafine particles in type lb boutons or type Is boutons in the early pupal stage (Fig. 3C-D).

Therefore, degeneration of NMJ boutons originates from swelling and retraction of the SSR membrane, and then, the clear synaptic vesicles turn into dark synaptic vesicles and fragment into dark ultrafine particles along with degeneration of the T-bar structure from the presynaptic membrane.

\section{$d n r x$ mutation leads to degeneration of NMJ boutons}

We analyzed $d n r x, d n l g 1, d n l g 2, d n l g 3$ and $d n l g 4$ single mutants and found that $d n r x^{273}$, the nrx null mutant, led to degeneration of NMJ boutons in Drosophila. The degenerating NMJ boutons in $d n r x^{273}$ flies (Fig. 4-5) demonstrated more significant degeneration than observed in wild-type Drosophila (Fig. 13). The terminal of degenerated NMJ boutons, without a T-bar structure or other organelles, was smaller than that of normal boutons (Fig. 4A) and was filled with dark ultrafine particles (Fig. 4A'-A") but lacked dark synaptic vesicles, whereas the adjacent type lb bouton was filled with clear vesicles (Fig. 4A', $A^{\prime \prime}$ ) and several dark synaptic vesicles (Fig. 4A). There was no SSR membrane between the degenerated bouton and the adjacent normal bouton (Fig. 4B), which also suggested that the SSR retracted severely as the NMJ bouton degenerated. The SSR membrane was sparse and loose near the degenerated bouton (Fig. $4 \mathrm{C}-\mathrm{C}^{\prime}$ ) but was relatively normal compared with the adjacent type Ib bouton (Fig. $4 \mathrm{C}$ and $\mathrm{C}^{\prime \prime}$ ). In the seriously degenerated boutons, the axon terminals showed signs of degeneration/vacuolization, and the SSR membrane had obviously swollen and withdrawn (Fig. 4A-A', C-C', and D-D'). It is worth noting that the contents of degenerated terminals in $d n r x^{273}$ mutants (Fig. 4A', $C^{\prime}$ and D-E) were much denser than those of wild-type terminals (Fig. 2C-C"). We observed another degenerated bouton that had an irregular morphology, a seriously linearized and degenerated SSR (Fig. 4E-E"), degenerated contents in the axon terminal, an obvious residual postsynaptic area (PSA) and a synapse with almost no synaptic cleft (Fig. $\left.4 \mathrm{E}^{\prime} \mathrm{E}^{\prime}\right)$. The residual PSA suggested that the NMJ bouton was degenerated in situ but not eliminated. The degeneration of NMJ boutons could originate from ghost boutons and developing boutons. The abnormal SSR phenotype could not be observed in ghost synapses due to their lack of an SSR. The appearance of large ghost boutons was irregular in $d n r x^{273}$ mutants (Fig. 4F-G), while normal ghost boutons in wild-type flies ([44]) and in some mutants [49] were spherical and full of clear vesicles. Instead of the clear and dark synaptic vesicles, there were dense dark ultrafine particles in the ghosts (Fig. 4F-G). However, some dark ultrafine particles were sparse (Fig. 4F-G'), and other dark ultrafine particles were intensively clustered (Fig. 4G, G"). A thin SSR membrane was occasionally visible (Fig. 4G'). The degeneration might originate from developing boutons in which there were dark ultrafine particles (Fig. $\left.4 \mathrm{H}, \mathrm{H}^{\prime}\right)$, degraded synapses with a thin presynaptic membrane and postsynaptic membrane (Fig. $\left.4 \mathrm{H}, \mathrm{H}^{\prime \prime}\right)$, and swollen and linear SSR membranes (Fig. $4 \mathrm{H}^{\prime}, \mathrm{H}^{\prime \prime}$ ).

Since mutant NMJ bouton degeneration was more serious in the $d n r x^{273}$ mutant, we suspected that it might be easier to observe the fine degeneration of synaptic vesicles in the mutant by utilizing TEM. As reported in the literature $[45,46]$, type lb boutons had clear synaptic vesicles with a T-bar (Fig. 5 A-A'), type Is had extremely sparse dense core vesicles (Fig. 5 B-B'), type II had more dense core vesicles (Fig. 5 C-C'), 
type III only included dense core vesicles (data not shown), and most clear synaptic vesicles were clustered with actin filaments (Fig. 5 A', $^{\prime} \mathrm{B}^{\prime}, \mathrm{C}^{\prime}$ ).

Before NMJ boutons were severely degenerated, the T-bars detached from the presynaptic membrane. In all degenerated boutons, we observed a residual synapse that only included the presynaptic membrane and postsynaptic membrane, but no presynaptic T-bar that recruits and docks synaptic vesicles was present. The T-bar (Fig. 5D) detached from the presynaptic membrane, and the shed T-bar clustered dense synaptic vesicles (Fig. 5D-D') [50], which might hinder accumulation of synaptic vesicles near the presynaptic membrane (Fig. 5D', Fig. 5E (shown in the black box)) and move them to the center of the NMJ bouton, but in the same type Ib bouton the peripheral synaptic vesicles gathered in another T-bar that looked as if it was about to detach from the presynaptic membrane.

Then, we observed two degeneration modes of synaptic vesicles in type lb boutons that could avoid the interference of dense core vesicles in type Is, type II and type III, according to the electron density under electron microscopy. In the first mode, one or two dark ultrafine spots occurred on the membrane of clear synaptic vesicles near a synapse with a relatively intact T-bar (Fig. 5F), and more dark ultrafine spots developed on the clear synaptic vesicle membrane and formed a circle at a site farther from the same synapse (Fig. 5F, F'). Before another synapse without a T-bar, the dark ultrafine spots dispersed into irregular profiles with larger sizes than the other synaptic vesicles (Fig. $5 \mathrm{G}^{\prime}$ ); therefore, we believe the irregular profiles were the result of collapse and dispersion from the degenerated synaptic vesicles with dark ultrafine spots, and two collapsed synaptic vesicles overlapped each other to form a large profile (Fig. 5 G, G'). The slightly collapsed vesicles had the appearance of an ellipsoid profile, with dark spots on the inside and outside and a size similar to clear vesicles (Fig. $5 \mathrm{G}$, lower right corner). It is worth noting that the clear synaptic vesicles away from the synapse had a tendency to detach from each other without actin filaments (Fig. $5 \mathrm{~F}^{\prime}, \mathrm{G}^{\prime}$ ). Therefore, the first mode of synaptic vesicle degeneration occurred on the membrane with ultrafine spots and showed a collapsed and dispersed irregular profile with dark ultrafine particles.

In the second mode, the clear synaptic vesicles degenerated into dense synaptic vesicles, formed irregular dark clumps, and collapsed and dispersed an irregular profile with dark ultrafine particles. The degenerating bouton, a type lb bouton in the $d n r x^{273}$ mutant with only clear synaptic vesicles $[45,46]$ (Fig. $5 \mathrm{H}$ ), had five synapses without T-bars and had numerous synaptic vesicles on its periphery. In high magnification mode, the synaptic vesicles could be divided into clear vesicles and different dense vesicles both with membranes near the periphery and cortex of an axon terminal (Fig. $\left.5 \mathrm{H}, \mathrm{H}^{\prime}\right)$, but the clear vesicles, dense vesicles, dark clumps without a membrane, and dark ultrafine particles were present in the center of the axon terminal (Fig. $\left.5 \mathrm{H}, \mathrm{H}^{\prime \prime}\right)$. The dense vesicles and clear vesicles could be clustered with actin filaments (Fig. $5 \mathrm{I}$ ) or detached without actin filaments (Fig. $5 \mathrm{~J}$ ), and they appeared to exhibit deepening electron density (Fig. $5 \mathrm{~K}-\mathrm{V}$ ). A short dark line occurred on a certain point on the membrane of clear vesicles (Fig. $5 \mathrm{~K}$ ), and the dark line expanded along the membrane of synaptic vesicles (Fig. $5 \mathrm{~L}$ ) until it covered the entire membrane (Fig. $5 \mathrm{M}-\mathrm{N}$ ), which made the vesicle dark. The electron density in vesicles expanded inward (Fig. 5 0), and the clear region in the dark vesicle continually decreased (Fig. 
5P-Q). Then, the vesicle became fully electron dense, the membrane and morphological profile of the vesicle were lost, and a dark clump without actin filaments formed (Fig. $5 \mathrm{R}$ ). The clump became darker (Fig. $5 \mathrm{~S}$ ) and separated into several dark ultrafine particles at the edge of the clump (Fig. $5 \mathrm{~T}$ ), and the number of ultrafine particles increased at the edge of the clump (Fig. $5 \mathrm{U}$ ) until many fragmented dark ultrafine particles were present (Fig. 5V), which could be regarded as direct evidence that the dark vesicle had broken into ultrafine particles. The dark clumps had different sizes due to the different vesicle sizes. Once many vesicles were adhered to each other with actin filaments and degenerated together, they formed a big clump of ultrafine particles (Fig. 4A', C', E', G"). Accordingly, it was easier to observe the dynamics of synaptic vesicle degeneration in $d n r x^{273}$ mutants, and the speckled membrane of clear vesicles, dark vesicles, dark clumps, and dark ultrafine particles could be regarded as signs of synaptic vesicle degeneration without lysosome involvement.

\section{$d n l g 1$ and $d n l g 4$ mutants exhibited NMJ bouton degeneration}

According to the signs of synaptic vesicle degeneration, we found that $d n \lg 1$ and $d n \lg 4$ mutants exhibited NMJ bouton degeneration. There was significant degeneration of axon terminals in NMJ boutons in $d n l g 1$ mutants, but the SSR remained relatively intact (Fig. 6A-A', C-C'). In the axon terminal, there were several plaques (Fig. 6A-A'), and the degenerated synaptic vesicles were in the plaques in the form of dark synaptic vesicles (Fig. 6A'-A', B-B') and dark ultrafine particles (Fig. 6B-B'), along with the clear synaptic vesicles and T-bar (Fig. 6A"). Therefore, plaques with dark synaptic vesicles and dark ultrafine particles could be seen as markers of synaptic degeneration.

Degeneration was accompanied by abnormal assembly of microtubules. We observed long microtubules protruding into a type $\mathrm{lb}$ bouton in both directions (from above and below) (Fig. 6C-C'), and the downward pointing microtubule passed through clear vesicles (Fig. $6 \mathrm{C}^{\prime}-\mathrm{C}^{\prime \prime}$ ) and reached a small plaque with a dark synaptic vesicle that showed degeneration (Fig. 6C"). Degeneration could occur in axons. White plaques occurred among microtubules in a large axon (Fig. 6D) and contained dark synaptic vesicles and dark ultrafine particles (Fig. 6D'-D"), but clear vesicles were not present in the white plaque (Fig. 6D', upper right corner). Furthermore, some axons of motor nerve fibers also showed degeneration in dnlg1 mutants (Fig. $6 \mathrm{C}, \mathrm{E}$ ). The degenerated axons contained dark ultrafine particles (Fig. 6E'-E') and gathered in specific parts of fibers (Fig. 6E). In the other part of the same fiber, the axon looked intact, with clear vesicles and microtubules pointing in different directions (Fig. 6E, F-F'). Therefore, the boundary between the degenerated axons and the normal axons could be artificially drawn (Fig. 6E).

The axon terminals also degenerated with SSR retraction (Fig. G) in NMJ boutons in dnlg4 mutants, and dark vesicles (Fig. 6G-G'), dark ultrafine particles and spare SSR membrane were observed (Fig. 6G, G"$\left.\mathrm{G}^{\prime \prime}\right)$. Interestingly, autophagy was found in degenerated axons (Fig. 6H-H').

\section{$d n l g 2$ and $d n l g 3$ coregulate synaptic footprints in Drosophila NMJs}

Both $d n \lg 2$ [41] and $d n \lg 3$ [42] regulate the circulation of synaptic vesicles, but dark vesicles and dark ultrafine particles were not observed in more than $30 \mathrm{NMJ}$ boutons in $d n \lg 2$ (Fig. 7A-A') and $d n \lg 3$ (Fig. 
7B-B') single mutants or $d n / g 2$ - and $d n l g 3$-overexpressing lines (data not shown). However, degeneration of NMJ boutons frequently occurred both in the axon terminal and SSR in $d n l g 2 ; d n / g 3$ double mutants. The degeneration primarily emerged in the center of the axonal terminal (Fig. 7C-C', C'”, E), with dark vesicles and dark ultrafine particles visible (Fig. $\left.7 \mathrm{C}^{\prime \prime}\right)$, but the clear synaptic vesicles were mainly distributed around the axonal membrane with presynaptic ruffles (Fig. 7C"). Moreover, the SSR was disordered (Fig. 7D-D') or even retracted to form a large PSA with the T-bar (Fig. 7E-E'). In outer motor nerve fibers, the degeneration of axons mainly emerged with dark vesicles and dark ultrafine particles (Fig. 7F, F"). However, in most axons of the same nerve fiber, the periphery of the axons was relatively intact, with clear vesicles inside ( $\left.F-F^{\prime}\right)$, and most axons inside the fiber $(F)$ looked ordered, without dark vesicles or dark ultrafine particles, which suggested that the peripheral axons of nerve fibers were more susceptible to degeneration. Interestingly, we found normal microtubules along with abnormal microtubules that had a smaller diameter and dark electron density in the small axon $\left(F^{\prime}\right)$.

\section{$d n r x$ and $d n l g 3$ colead degeneration in Drosophila NMJs}

$d n r x^{83}$ and $d n r x^{174}$ are hypomorphic mutants [38], and they live to adulthood, while $d n r x^{273}$ is a null mutant [39], which is lethal during the pupa stage. Under electron microscopy, $d n r x^{273}$ mutants had severe degeneration in NMJ boutons (Fig. 4-5), but the $d n r x^{83 / 174}$ mutant did not show a degeneration phenotype. In addition, SSR degeneration and obvious dark vesicles were not observed in axon terminals in $d n r x^{83}$ (data not shown), $d n r x^{174}$ (data not shown), $d n r x^{83 / 174}$ (Fig. 8A-A'), and $d n / g 3$ (Fig. 7B-B', Fig. 8B$\left.\mathrm{B}^{\prime \prime}\right)$ mutants. However, NMJ bouton degeneration occurred in both the axon terminal and SSR of the $d n r x 83 ; d n l g 3$ double mutant (Fig. 8C-E'). We observed dark vesicles in the axon terminal of $d n r x 83 ; d n l g 3$ double mutants (Fig. 8C-C'). The SSR retracted and formed a rare SSR membrane (Fig. 8C, C"), and a portion of the SSR was disordered (Fig. 8C, C'"). The sparse SSR membrane could form a large PSA outside of NMJs (Fig. 8C). The T-bar structure was detached from the presynaptic membrane with clustered synaptic vesicles (Fig. 8 D-D'), and several dark lysosomes were observed in the PSA that was near the postsynaptic membrane (Fig. 8 D, D"). A degenerating type Is bouton was observed that had almost had no SSR membrane and contained a T-bar, dark vesicles (Fig. 8 E-E'), and myelin-like mitochondria that were severely damaged (Fig. 8 E, E"). The type lb bouton had more large-sized clear vesicles in the $d n r x^{83 / 174}$ (Fig. 8A-A') and $d n / g 3$ (Fig. 7B-B', Fig. 8B-B") mutants, and the large clear vesicles further increased and collapsed inwardly in the $d n r x 83 ; d n l g 3$ double mutant (Fig. $\left.8 \mathrm{C}-\mathrm{C}^{\prime}, \mathrm{C}^{\prime \prime \prime}\right)$.

\section{Synaptotagmin is not distributed in ultrafine particles in degenerated boutons}

Synaptotagmin (Syt) and synapsin (Syn) are synaptic vesicular proteins and have been used as markers of synaptic vesicles in Drosophila in many studies. Based on our above results, we propose a scenario for synaptic vesicle degeneration: spherical clear synaptic vesicles collapse into dark vesicles and then fragment into 2-3 nm ultrafine particles. Syt was present in the NMJ bouton in wild-type flies under light microscopy (Fig. 9 A-A'), Syt (Fig. 9 B-C') and Syn (Fig. 9 D-D') were present in the synaptic vesicles in TEM, but Syt and Syn were not present in the control (Fig. 9 E-E') evaluated with preembedding immunogold electron microscopy. 
Due to the obvious degeneration of NMJ boutons and presence of ultrafine particles in the $d n \lg 2 ; \mathrm{dn} / g 3$ double mutants, we investigated whether Syt was present in these ultrafine particles. Syt was present in synaptic vesicles of NMJ boutons without ultrafine particles (Fig. 9F). However, Syt was not present in the ultrafine particles but was present in the synaptic vesicles in the degenerated NMJ boutons (Fig. 9G-G"). Therefore, in the process of synaptic vesicle degeneration into ultrafine particles, synaptic vesicleassociated proteins, such as Syt, appear to be completely degraded and could not be detected by the corresponding antibodies in TEM.

\section{Neurexin and neuroligins jointly regulate synapse degeneration at the neuromuscular junction}

Syt was not observed via TEM in the ultrafine particles that were degeneration products, which indicates that the degenerated synaptic vesicles might lose the signaling of Syt and Syn proteins with the disintegration of synaptic vesicles. Next, we investigated whether the degenerated NMJ boutons could be observed via confocal microscopy with a 3D scanning function for biological samples. To facilitate the evaluation of degenerated NMJ boutons, we observed and counted type lb boutons that had a larger size, and the synaptic vesicles were numerous and relatively dispersed in the outer layer of axon terminals with respect to the type Is boutons $[45,46]$.

Most type lb boutons had strong Syt (data not shown) and Syn (Fig. 10 A-A") protein signals at the 6th/7th muscles in the $A_{3}$ or $A_{2}$ segment in wild-type lines. The Syn signals in type lb boutons were regular, globular and covered the entire axon terminal in large pinhole mode (We adjusted the pinhole to 600 to acquire thicker optical sections with a highly sensitive GaAsP detector and used an 80 pinhole for routine observation.), and few degenerated type lb boutons were observed $(0.63 \pm 0.26, \mathrm{~N}=8)$. After the confocal microscopy focal length was adjusted, although speckled Syn signals were present at some optical sections, in the middle optical area, 2-3 sections were always filled with Syn signals in axon terminals (Fig. $10 \mathrm{~A}-\mathrm{A}^{\prime \prime}$ ). There were very weak or no Syn signals in some type ls boutons under the same microscopy parameters, including pinhole size, laser intensity and image brightness.

However, several degenerated type Ib boutons could be observed in $d n r x^{273}(2.25 \pm 0.25, \mathrm{~N}=4)$ (Fig. $10 \mathrm{~B}$ B"), $d n \lg 1(3.29 \pm 0.75, \mathrm{~N}=7)$ (Fig. $\left.10 \mathrm{C}-\mathrm{C}^{\prime \prime}\right)$, and $d n \lg 4(2.63 \pm 0.53, \mathrm{~N}=8)$ (Fig. $10 \mathrm{~F}-\mathrm{F}$ ) mutants. The criteria for judging the degenerated type lb boutons were as follows: 1 . The Syn signals were always very weak compared with those in other type Ib boutons (Fig. 10 B-B"); 2. The Syn signals were always distributed in spots in the bouton (Fig. 10 C-C", F-F"); 3. After adjustment of the confocal microscope pinhole size and laser intensity and the image brightness, the Syn signals in degenerate type lb boutons faded (Fig. 10 B-B") or were distributed in small dots (Fig. 10 G-G") in most instances, but the Syn signals remained spherical and dense in other type $\mathrm{lb}$ boutons. There were not obvious degenerate type $\mathrm{lb}$ boutons in $d n \lg 2(1.33 \pm 0.33, \mathrm{~N}=6)$ (Fig. $10 \mathrm{D}-\mathrm{D} ")$, dnlg3 $(0.53 \pm 0.14, \mathrm{~N}=13)$ (Fig. $\left.10 \mathrm{E}-\mathrm{E}^{\prime \prime}\right)$, and $d n r x^{83}$ $(0.88 \pm 0.30, \mathrm{~N}=8)$ mutants (Fig. $\left.10 \mathrm{I}-\mathrm{I}^{\prime}\right)$, which were partial mutants of whole $d n r x$ genes. Interestingly, the degenerate type $\mathrm{lb}$ boutons were frequently found in $d n \lg 2 ; \mathrm{dn} / g 3(4.75 \pm 0.85, \mathrm{~N}=4)$ (Fig. $10 \mathrm{G}-\mathrm{G}$ ") and $d n r x^{83}$; $\ln \lg 3(3.13 \pm 0.35, \mathrm{~N}=8)$ double mutants (Fig. $\left.10 \mathrm{H}-\mathrm{H}^{\prime \prime}\right)\left(\mathrm{Fig} .10 \mathrm{I}-\mathrm{I}^{\prime \prime}\right)$. Therefore, the synaptic vesicle- 
associated protein Syn could not be detected with the corresponding antibodies via confocal microscopy, and the degeneration of terminals was accelerated in $d n r x$ and $d n l g s$ mutants.

\section{Discussion}

\section{Ultrastructural features of NMJ bouton degeneration in third instar Drosophila larva}

Synaptic degeneration can be caused by degenerative neurological diseases, such as Alzheimer's disease $[51,52]$ and prion disease $[13,24]$; may also be caused by injuries, such as surgery, microwaves [20] and mild fluid percussion [53]; or can occur in aging animals [15,22]. Fragmentation and degeneration of organelles exposes more proteins or polypeptides, which contain amino groups to which osmic acid can easily adsorb, resulting in electron density under an electron microscope. According to the current literature $[21,51,54]$, degeneration of neuronal cytoplasm and synaptic boutons is characterized by dark electron density under an electron microscope.

The Drosophila larval NMJ is a powerful experimental model, and it contains three bouton types: type I, type II and type III boutons. Type I boutons are often used for studying synaptic development, signal transmission and neurological disease and are repeatedly wrapped by the subsynaptic reticulum (SSR) that is formed by the muscle cell membrane, and the synapse, T-bar structures, synaptic vesicles, mitochondria, and cytoskeleton appear in the axon terminal. Type I boutons include type lb (big) and type Is (small). At present, ultrastructural phenotype analysis of type I boutons has mainly focused on T-bars, synaptic vesicles and the SSR [42, 43], and few studies ([44]) have analyzed ghost boutons, which are indicators of poor bouton development; hypogenetic boutons; or satellite boutons, which are indicators of synaptic overgrowth. Electron microscopy is a powerful tool to study synapse structure [55]. In the present study, we describe the ultrastructural characteristics of Drosophila larval NMJ bouton degeneration, primarily based on the dark electron density observed via electron microscopy.

The degeneration of Drosophila NMJ boutons included collapse and fragmentation of synaptic vesicles, retraction and degradation of the SSR, and deformation of the profile. The normal synaptic vesicles were globular, clear, and small (approximately $35 \mathrm{~nm}$ diameter), with a single membrane layer. The clear synaptic vesicles collapsed into dark synaptic vesicles and then fragmented into ultrafine particles during the process of terminal degeneration. We deduced that this process involves the following steps: globular, clear synaptic vesicles collapse out or in and form an irregular, bigger or smaller profile with membrane laceration, which allows lipids and proteins in vesicles be fully exposed and easily stained by heavy metals, such as osmium, acetic acid and lead citrate; thus, the dark synaptic vesicles were electron dense under an electron microscope. Then, the dark synaptic vesicles are further degraded and fragmented into ultrafine particles. Neuro-filament bundles accumulate in the degenerating neuronal cytoplasm $[13,15]$, similar to lysosome accumulation [13] and neuro-filament degeneration in injured brains [21]. We observed intrusion of disturbed microtubules into NMJ boutons where actin and synaptic vesicles should be located, and dark synaptic vesicles formed at the end of microtubules in the boutons. In $d n \lg 1$ mutants, the dark synaptic vesicles also occurred along the long axon. Therefore, the fragmentation of 
synaptic vesicles was associated with abnormal assembly and transport of microtubules. Furthermore, the NMJ boutons contained autophagosomes (Fig. 6H-H') [54] and abnormal mitochondria (Fig. 8E, E"), which are associated with synaptic degeneration [13]. NMJ boutons also contained presynaptic degradation products, including presynaptic organelle components, cytoskeleton components and T-bar components, degenerated together and gathered in dark clumps (Fig. 4A-A") in the axon terminal, and we were unable to completely distinguish the morphological structure of organelles [24].

Presynaptic organelles appear swollen or dark and dense [21], and degenerating dendrites are also dark [51] during synapse degeneration. Once the synapse is degenerated, the PSD looks curved [24], and both presynaptic and postsynaptic membranes have been shown to become thin in aging mice [22] (Fig. 1GG"). During NMJ bouton degeneration in wild-type Drosophila, the complex SSR membrane became swollen and retracted until a few remnants were left, but more SSR remnants degenerated into fragments or thin slices in dnrx mutants (Fig. 4-5). Degeneration of NMJ boutons starts from the SSR membrane, but the rate of SSR membrane degeneration is slower than that of the axon terminal. Degeneration of SSR membranes primarily manifested as retraction, and synaptic vesicles and presynaptic organelles primarily showed lysis and collapse.

With degeneration of the presynaptic terminal and postsynaptic SSRs, NMJ boutons lost their globular spherical profile and become irregular, with a small profile.

The degenerated synaptic boutons are engulfed by Schwann cells [14-17] in mammalian NMJs, but degenerated NMJ boutons appear to not be eliminated by glial cells or muscle cells in Drosophila. Therefore, degenerated NMJ boutons degenerate in situ and appear to be abandoned in the muscles because most of the organs, including muscles and the NMJ system, will be completely autolyzed in the next pupal stage.

\section{NMJ bouton development or degeneration: Ultrastructural differences}

The number of NMJ boutons increases 10-fold from the first instar to third instar in Drosophila [56], and degenerated NMJ boutons can be seen as remnants of the pruned NMJ boutons during the process of NMJ bouton development. The degenerated NMJ boutons were rare in wild-type flies, which meant that most pruned NMJ boutons produced physiological retraction, firming the synaptic footprint [30, 31].

Axon terminals were small, synaptic vesicles were dark, and postsynaptic SSRs were loose and thin in degenerating NMJ boutons, which happens to be a feature of developing NMJ boutons ([44]). Thus, how can degenerating and developing NMJ boutons be distinguished? In degenerating NMJ boutons, the speckled membrane of clear vesicles, dark vesicles and dark clumps without a membrane, dark ultrafine particles, synaptic vesicles and mitochondria were reduced; the T-bar was detached from the presynaptic membrane; and mitochondria were swollen, with few and fractured cristae [21, 22]. Furthermore, the SSR was reduced and irregularly disorganized, with a large PSA. In developing NMJ boutons, the dark synaptic vesicles are spherical and wrapped in a complete biofilm [57]. 


\section{DNrx and DNlgs cause NMJ bouton degeneration}

The synaptic adhesion molecule neurexin (Nrx) is mainly located in the presynaptic membrane, and neuroligin ( $\mathrm{Nlg}$ ) is mainly distributed in the postsynaptic membrane. Nrx and $\mathrm{Nlg}$ defects lead to autism and neurological disorders. There is $1 d n r x$ gene and $4 d n / g$ genes in Drosophila, and the current studies on $d n r x[39,58]$ and $d n l g 1-4[40]$ have focused on synaptic signaling and synapse development $([44])$ in NMJ boutons.

Here, our results showed that $d n r x$ and $d n l g s$ caused NMJ bouton degeneration in Drosophila. Neurodegenerative diseases are accompanied by severe synaptic degeneration [21, 51, 52]. The NMJ boutons showed severe degeneration in the $d n r x^{273}, d n l g 1$, and $d n l g 4$ mutants, but there was no obvious synaptic degeneration in the $d n r x^{83}, d n l g 2$ and $d n l g 3$ mutants. However, there was obvious synaptic degeneration in the $d n l g 2 ; d n l g 3$ and $d n r x^{83} / d n l g 2$ double mutants, which further confirms that there is a synergistic function between $d n r x$ and $d n \operatorname{lgs}$ and between $d n \lg 2$ and $d n \lg 3$.

$d n r x$ and $d n$ lgs mutation interfered with the BMP and Wnt signaling pathways by disrupting spectin [59], actin [58], and microtubules [60], all of which are essential cytoskeleton components necessary for synapse formation and development. Mutations in members of the BMP pathway, such as wit and $g b b$ $[50,61]$, have been shown to cause abnormal presynaptic ruffles that were similar to those observed in $d n r x$ and $d n l g s$ mutants $[40,43,60]$ and caused presynaptic shedding of T-bars [50], similar to that observed in $d n r x^{273}$ mutants (Fig. 5) via TEM. The $d n l g 4$ gene and other gene members of the BMP pathway, such as wit, tkv, and mad, have been found to have a dose-dependent genetic interaction in NMJ development [43].

Wnt signaling is involved in regulation of synaptic morphology and functional plasticity, and Wnt deficiency is closely related to Alzheimer's disease [62]. Blocking the secretion of Wg (Wingless, a Wnt homologue in flies) at a certain stage of development inhibits the growth of boutons by destroying the presynaptic microtubule skeleton [49]. We also found abnormal microtubule skeletons in $d n / g 1$ mutants (Fig. 6C), which could transport other organelles, and microtubules in dnrx mutants have been shown to be broken [60]. Recent studies have shown that Nrx and Nlgs defects cause or worsen neurodegenerative diseases, and the expression levels of $\mathrm{Nlg}$ and $\mathrm{Nrx}$ are significantly downregulated in Alzheimer's disease $[63,64]$, which suggests that Nrx and Nlg are associated with the synaptic degeneration that occurs in Drosophila NMJ boutons.

Therefore, in studying the autism caused by $n r x s$ and $n l g s$, attention should be given not only to research on classic synaptic signal transmission and on the developmental balance of synaptic boutons ([44]) but also to synaptic degeneration, which could also cause abnormal synaptic signal transmission.

\section{NMJ bouton degeneration patterns}

According to our findings and the current literature, we propose a model of NMJ bouton degeneration (Fig. 11). Type Ib boutons contain T-bars, dense clear synaptic vesicles, mitochondria, and a regular SSR 
with a PSA (Fig. 11 A). After NMJ bouton degeneration, SSR membranes begin to swell and retract, and some organelles, such as mitochondria and synaptic vesicles, are transported away from the terminal and reduced (Fig. 11B) [22], accompanied by reduction and fading of the presynaptic cytoskeletonassociated molecule Futsch [32,33] from distal boutons, which causes presynaptic contraction of the synapse and forms a synaptic footprint that has a smaller axon terminal and fewer synaptic vesicle signals [30, 31]. Then, the T-bar detaches from the presynaptic membrane [50] with the swelling of mitochondria [20], some degenerating dark vesicles appear in the center of terminals, and the SSR further swells and retracts (Fig. $11 \mathrm{C}$ ). All the T-bars detach from the presynaptic membrane and cluster the clear synaptic vesicles, more dark vesicles deform and collapse into irregular dark vesicles without a biofilm and then fragment into dark ultrafine particles (Fig. 1-2), and the SSR becomes more retracted and fluffy, along with PSA expansion or reduction (Fig. 11 D). Then, the terminal deforms, with more collapsed dark vesicles and residual synaptic vesicles, mitochondria and PSA, and most of the SSR becomes loose and disordered, some of which degenerates into a linear morphology (Fig. $11 \mathrm{E}$ ). Last, all residual membranes, including presynaptic and postsynaptic membranes [22], become very thin; most of the SSR degenerates into a linear shape; and all the residual elements in axon terminals, such as synaptic vesicles, mitochondria, cytoskeleton components, and T-bars, degenerate in situ and eventually form a cluster of ultrafine particles (Fig. 11 F).

Axon terminals and elements within axon terminals degenerate with the postsynaptic SSR. However, swelling and retraction of the SSR occurs prior to axon terminal degeneration, which is faster and more intense than SSR degeneration. Furthermore, the degeneration of synaptic vesicles begins at the center of terminals and follows two specific degeneration procedures (Fig. 11G).

NMJ bouton degeneration occurs under normal physiological conditions but is accelerated in dnrx and $d n l g s$ mutants. Furthermore, there is a synergistic effect in $d n r x$, $d n l g s$ double mutants and $d n \lg 2 ; d n l g 3$ double mutants that promotes degeneration of NMJ boutons.

\section{Conclusions}

NMJ bouton degeneration occurs under normal physiological conditions but is accelerated in dnrx and $d n l g s$ mutants. Furthermore, there is a synergistic effect in $d n r x$, $d n \lg s$ double mutants and $d n \lg 2 ; d n \lg 3$ double mutants that promotes degeneration of NMJ boutons, which suggests that both neurexin and neuroligins play a vital role in preventing synaptic degeneration. This study proposes the model of NMJ bouton degeneration patterns, which is very conducive to in-depth study of neurodegeneration.

\section{Abbreviations}

NMJ: neuromuscular junction

SSR: subsynaptic reticulum

PSA: postsynaptic area 
dnrx. dneurexin, Drosophila neurexin,

dngl: dneuroligin, Drosophila neuroligin

TEM: transmission electron microscopy

BMP: bonemorphogenetic protein

Wnt: wingless-int

\section{Declarations}

\section{Ethics approval and Consent to participate}

The research involved animals, which were all bred in the animal facility at Southeast University. All experiments were performed according to guidelines approved by Southeast University, and no informed consent was required for this research. All authors had full access to all data in the study and take responsibility for the integrity of the data and the accuracy of the data analysis.

\section{Consent for publication}

All authors agree to submit to your journal and know the ethical rules of submission.

\section{Availability of data and materials}

The data generated during this study is included in this published article [and its Additional file 1: Figure S1-S4].

\section{Competing interests}

The authors declare no competing financial interests.

\section{Funding}

This work was supported by the Southeast University Fundamental Research Fund (3224005416), Southeast University Analysis Test Fund (11240090971), and Student Research Training Program (201810286128) of Southeast University, Nanjing, China.

\section{Authors' contributions}

Study concept and design: G.G. and X.W. Acquisition of the data: G.G., G.J., and G.X. Analysis and interpretation of the data: G.G., and G.J. Drafting of the manuscript: G.G. and Z.C. Critical revision of the manuscript for important intellectual content: G.G. and Z.C. Statistical analysis: G.G. Funding: G.G and G.X. Administrative, technical, and material support: G.G., L.K., and Z.C. Study supervision: G.G and G. J. All authors read and approved the final manuscript. 
Acknowledgments

We are grateful for the Electron Microscopy Laboratory, School of Medicine, Southeast University, Nanjing, which provided all the data.

\section{Authors' information}

Gan Guangming ${ }^{1,2}$, Zhang Chenchen ${ }^{1,2}$, Gao Xiang ${ }^{1}$, Lu Keze ${ }^{1}$, Xie Wei ${ }^{2,3}$, Geng Junhua ${ }^{2}$

1. School of Medicine, Southeast University, Nanjing, Jiangsu 210009, China.

2. School of Life Science and Technology, the Key Laboratory of Developmental Genes and Human Disease, Southeast University, Nanjing, Jiangsu 210009, 210009, China.

3. School of Life Science and Technology, the Collaborative Innovation Center for Brain Science, Southeast University, Nanjing, Jiangsu 210009, China.

Correspondence: Guangming Gan and Junhua Geng

\section{References}

1. Watts RJ, Schuldiner O, Perrino J, Larsen C, Luo L: Glia engulf degenerating axons during developmental axon pruning. Curr Biol 2004, 14:678-684.

2. Awasaki T, Tatsumi R, Takahashi K, Arai K, Nakanishi Y, Ueda R, Ito K: Essential role of the apoptotic cell engulfment genes draper and ced-6 in programmed axon pruning during Drosophila metamorphosis. Neuron 2006, 50:855-867.

3. Lu TY, MacDonald JM, Neukomm LJ, Sheehan AE, Bradshaw R, Logan MA, Freeman MR: Axon degeneration induces glial responses through Draper-TRAF4-JNK signalling. Nat Commun 2017, 8:14355.

4. Perry S, Han Y, Das A, Dickman D: Homeostatic plasticity can be induced and expressed to restore synaptic strength at neuromuscular junctions undergoing ALS-related degeneration. Hum Mol Genet 2017, 26:4153-4167.

5. Cao Y, Chtarbanova S, Petersen AJ, Ganetzky B: Dnr1 mutations cause neurodegeneration in Drosophila by activating the innate immune response in the brain. Proc Natl Acad Sci U S A 2013, 110:E1752-1760.

6. Siskova Z, Mahad DJ, Pudney C, Campbell G, Cadogan M, Asuni A, O'Connor V, Perry VH: Morphological and functional abnormalities in mitochondria associated with synaptic degeneration in prion disease. Am J Pathol 2010, 177:1411-1421.

7. Wilkerson JR, Tsai NP, Maksimova MA, Wu H, Cabalo NP, Loerwald KW, Dictenberg JB, Gibson JR, Huber KM: A role for dendritic mGluR5-mediated local translation of Arc/Arg3.1 in MEF2-dependent synapse elimination. Cell Rep 2014, 7:1589-1600. 
8. Mikuni T, Uesaka N, Okuno H, Hirai H, Deisseroth $\mathrm{K}, \mathrm{Bito} \mathrm{H}$, Kano M: Arc/Arg3.1 is a postsynaptic mediator of activity-dependent synapse elimination in the developing cerebellum. Neuron 2013, 78:1024-1035.

9. Chung WS, Clarke LE, Wang GX, Stafford BK, Sher A, Chakraborty C, Joung J, Foo LC, Thompson A, Chen C, et al: Astrocytes mediate synapse elimination through MEGF10 and MERTK pathways. Nature 2013, 504:394-400.

10. Yang J, Yang H, Liu Y, Li X, Qin L, Lou H, Duan S, Wang H: Astrocytes contribute to synapse elimination via type 2 inositol 1,4,5-trisphosphate receptor-dependent release of ATP. Elife 2016, 5:e15043.

11. Tasdemir-Yilmaz OE, Freeman MR: Astrocytes engage unique molecular programs to engulf pruned neuronal debris from distinct subsets of neurons. Genes Dev 2014, 28:20-33.

12. Miyamoto A, Wake H, Ishikawa AW, Eto K, Shibata K, Murakoshi H, Koizumi S, Moorhouse AJ, Yoshimura $Y$, Nabekura J: Microglia contact induces synapse formation in developing somatosensory cortex. Nat Commun 2016, 7:12540.

13. Siskova Z, Page A, O'Connor V, Perry VH: Degenerating synaptic boutons in prion disease: microglia activation without synaptic stripping. Am J Pathol 2009, 175:1610-1621.

14. Smith IW, Mikesh M, Lee Y, Thompson WJ: Terminal Schwann cells participate in the competition underlying neuromuscular synapse elimination. J Neurosci 2013, 33:17724-17736.

15. Gillingwater TH, Thomson D, Mack TG, Soffin EM, Mattison RJ, Coleman MP, Ribchester RR: Agedependent synapse withdrawal at axotomised neuromuscular junctions in Wld(s) mutant and Ube4b/Nmnat transgenic mice. J Physiol 2002, 543:739-755.

16. Lee YI, Li Y, Mikesh M, Smith I, Nave KA, Schwab MH, Thompson WJ: Neuregulin1 displayed on motor axons regulates terminal Schwann cell-mediated synapse elimination at developing neuromuscular junctions. Proc Natl Acad Sci U S A 2016, 113:E479-487.

17. Jung JH, Smith I, Mikesh M: Terminal Schwann cell and vacant site mediated synapse elimination at developing neuromuscular junctions. Sci Rep 2019, 9:18594.

18. Watts RJ, Hoopfer ED, Luo L: Axon pruning during Drosophila metamorphosis: evidence for local degeneration and requirement of the ubiquitin-proteasome system. Neuron 2003, 38:871-885.

19. Pielage J, Bulat V, Zuchero JB, Fetter RD, Davis GW: Hts/Adducin controls synaptic elaboration and elimination. Neuron 2011, 69:1114-1131.

20. Tan S, Wang H, Xu X, Zhao L, Zhang J, Dong J, Yao B, Wang H, Zhou H, Gao Y, Peng R: Study on dose-dependent, frequency-dependent, and accumulative effects of $1.5 \mathrm{GHz}$ and $2.856 \mathrm{GHz}$ microwave on cognitive functions in Wistar rats. Sci Rep 2017, 7:10781.

21. Qi L, Chen Z, Wang Y, Liu X, Liu X, Ke L, Zheng Z, Lin X, Zhou Y, Wu L, Liu L: Subcutaneous liraglutide ameliorates methylglyoxal-induced Alzheimer-like tau pathology and cognitive impairment by modulating tau hyperphosphorylation and glycogen synthase kinase-3beta. Am J Trans/ Res 2017, 9:247-260. 
22. Fan WJ, Yan MC, Wang L, Sun YZ, Deng JB, Deng JX: Synaptic aging disrupts synaptic morphology and function in cerebellar Purkinje cells. Neural Regen Res 2018, 13:1019-1025.

23. Pareek G, Thomas RE, Pallanck LJ: Loss of the Drosophila m-AAA mitochondrial protease paraplegin results in mitochondrial dysfunction, shortened lifespan, and neuronal and muscular degeneration. Cell Death Dis 2018, 9:304.

24. Caleo M, Restani L, Vannini E, Siskova Z, Al-Malki H, Morgan R, O'Connor V, Perry VH: The role of activity in synaptic degeneration in a protein misfolding disease, prion disease. PLoS One 2012, 7:e41182.

25. Tang G, Gudsnuk K, Kuo SH, Cotrina ML, Rosoklija G, Sosunov A, Sonders MS, Kanter E, Castagna C, Yamamoto $A$, et al: Loss of mTOR-dependent macroautophagy causes autistic-like synaptic pruning deficits. Neuron 2014, 83:1131-1143.

26. Frey D, Schneider C, Xu L, Borg J, Spooren W, Caroni P: Early and selective loss of neuromuscular synapse subtypes with low sprouting competence in motoneuron diseases. $J$ Neurosci 2000 , 20:2534-2542.

27. Fischer LR, Culver DG, Tennant P, Davis AA, Wang M, Castellano-Sanchez A, Khan J, Polak MA, Glass JD: Amyotrophic lateral sclerosis is a distal axonopathy: evidence in mice and man. Exp Neurol 2004, 185:232-240.

28. Fuentes-Medel Y, Logan MA, Ashley J, Ataman B, Budnik V, Freeman MR: Glia and muscle sculpt neuromuscular arbors by engulfing destabilized synaptic boutons and shed presynaptic debris. PLOS Biol 2009, 7:e1000184.

29. Sutcliffe B, Forero MG, Zhu B, Robinson IM, Hidalgo A: Neuron-type specific functions of DNT1, DNT2 and Spz at the Drosophila neuromuscular junction. PLoS One 2013, 8:e75902.

30. Eaton BA, Fetter RD, Davis GW: Dynactin is necessary for synapse stabilization. Neuron 2002, 34:729-741.

31. Eaton BA, Davis GW: LIM Kinase1 controls synaptic stability downstream of the type II BMP receptor. Neuron 2005, 47:695-708.

32. Xiong $X$, Collins CA: A conditioning lesion protects axons from degeneration via the Wallenda/DLK MAP kinase signaling cascade. J Neurosci 2012, 32:610-615.

33. Keller LC, Cheng L, Locke CJ, Muller M, Fetter RD, Davis GW: Glial-derived prodegenerative signaling in the Drosophila neuromuscular system. Neuron 2011, 72:760-775.

34. Ullrich B, Ushkaryov YA, Sudhof TC: Cartography of neurexins: more than 1000 isoforms generated by alternative splicing and expressed in distinct subsets of neurons. Neuron 1995, 14:497-507.

35. Zhang P, Lu H, Peixoto RT, Pines MK, Ge Y, Oku S, Siddiqui TJ, Xie Y, Wu W, Archer-Hartmann S, et al: Heparan Sulfate Organizes Neuronal Synapses through Neurexin Partnerships. Cel/ 2018, 174:14501464 e1423.

36. Polepalli JS, Wu H, Goswami D, Halpern CH, Sudhof TC, Malenka RC: Modulation of excitation on parvalbumin interneurons by neuroligin-3 regulates the hippocampal network. Nat Neurosci 2017, 20:219-229. 
37. Wu X, Morishita WK, Riley AM, Hale WD, Sudhof TC, Malenka RC: Neuroligin-1 Signaling Controls LTP and NMDA Receptors by Distinct Molecular Pathways. Neuron 2019, 102:621-635 e623.

38. Zeng X, Sun M, Liu L, Chen F, Wei L, Xie W: Neurexin-1 is required for synapse formation and larvae associative learning in Drosophila. FEBS Lett 2007, 581:2509-2516.

39. Li J, Ashley J, Budnik V, Bhat MA: Crucial role of Drosophila neurexin in proper active zone apposition to postsynaptic densities, synaptic growth, and synaptic transmission. Neuron 2007, 55:741-755.

40. Banovic D, Khorramshahi O, Owald D, Wichmann C, Riedt T, Fouquet W, Tian R, Sigrist SJ, Aberle H: Drosophila neuroligin 1 promotes growth and postsynaptic differentiation at glutamatergic neuromuscular junctions. Neuron 2010, 66:724-738.

41. Sun M, Xing G, Yuan L, Gan G, Knight D, With SI, He C, Han J, Zeng X, Fang M, et al: Neuroligin 2 is required for synapse development and function at the Drosophila neuromuscular junction. $J$ Neurosci 2011, 31:687-699.

42. Xing G, Gan G, Chen D, Sun M, Yi J, Lv H, Han J, Xie W: Drosophila neuroligin3 regulates neuromuscular junction development and synaptic differentiation. J Biol Chem 2014, 289:3186731877.

43. Zhang X, Rui M, Gan G, Huang C, Yi J, Lv H, Xie W: Neuroligin 4 regulates synaptic growth via the bone morphogenetic protein (BMP) signaling pathway at the Drosophila neuromuscular junction. $J$ Biol Chem 2017, 292:17991-18005.

44. Gan G, Zhang C: The precise subcellular localization of Dlg in the Drosophila larva body wall using improved preembedding immuno-EM. J Neurosci Res 2018, 96:467-480.

45. Atwood HL, Govind CK, Wu CF: Differential ultrastructure of synaptic terminals on ventral longitudinal abdominal muscles in Drosophila larvae. J Neurobiol 1993, 24:1008-1024.

46. Jia XX, Gorczyca M, Budnik V: Ultrastructure of neuromuscular junctions in Drosophila: comparison of wild type and mutants with increased excitability. J Neurobio/1993, 24:1025-1044.

47. Chen YC, Lin YQ, Banerjee S, Venken K, Li J, Ismat A, Chen K, Duraine L, Bellen HJ, Bhat MA: Drosophila neuroligin 2 is required presynaptically and postsynaptically for proper synaptic differentiation and synaptic transmission. J Neurosci 2012, 32:16018-16030.

48. Liu Z, Chen Y, Wang D, Wang S, Zhang YQ: Distinct presynaptic and postsynaptic dismantling processes of Drosophila neuromuscular junctions during metamorphosis. J Neurosci 2010, 30:11624-11634.

49. Packard M, Koo ES, Gorczyca M, Sharpe J, Cumberledge S, Budnik V: The Drosophila Wnt, wingless, provides an essential signal for pre- and postsynaptic differentiation. Cell 2002, 111:319-330.

50. Aberle H, Haghighi AP, Fetter RD, McCabe BD, Magalhaes TR, Goodman CS: wishful thinking encodes a BMP type II receptor that regulates synaptic growth in Drosophila. Neuron 2002, 33:545-558.

51. Jaworski T, Lechat B, Demedts D, Gielis L, Devijver H, Borghgraef P, Duimel H, Verheyen F, Kugler S, Van Leuven F: Dendritic degeneration, neurovascular defects, and inflammation precede neuronal loss in a mouse model for tau-mediated neurodegeneration. Am J Pathol 2011, 179:2001-2015. 
52. Dominguez-Alvaro M, Montero-Crespo M, Blazquez-Llorca L, Insausti R, DeFelipe J, Alonso-Nanclares $\mathrm{L}$ : Three-dimensional analysis of synapses in the transentorhinal cortex of Alzheimer's disease patients. Acta Neuropathol Commun 2018, 6:20.

53. Powell MA, Black RT, Smith TL, Reeves TM, Phillips LL: Mild Fluid Percussion Injury Induces Diffuse Axonal Damage and Reactive Synaptic Plasticity in the Mouse Olfactory Bulb. Neuroscience 2018, 371:106-118.

54. Li Q, Weiland A, Chen X, Lan X, Han X, Durham F, Liu X, Wan J, Ziai WC, Hanley DF, Wang J: Ultrastructural Characteristics of Neuronal Death and White Matter Injury in Mouse Brain Tissues After Intracerebral Hemorrhage: Coexistence of Ferroptosis, Autophagy, and Necrosis. Front Neurol 2018, 9:581.

55. Glausier JR, Konanur A, Lewis DA: Factors Affecting Ultrastructural Quality in the Prefrontal Cortex of the Postmortem Human Brain. J Histochem Cytochem 2019, 67:185-202.

56. Schuster CM, Davis GW, Fetter RD, Goodman CS: Genetic dissection of structural and functional components of synaptic plasticity. II. Fasciclin II controls presynaptic structural plasticity. Neuron 1996, 17:655-667.

57. Gan G, Lv H, Xie W: Morphological identification and development of neurite in Drosophila ventral nerve cord neuropil. PLoS One 2014, 9:e105497.

58. Rui M, Qian J, Liu L, Cai Y, Lv H, Han J, Jia Z, Xie W: The neuronal protein Neurexin directly interacts with the Scribble-Pix complex to stimulate F-actin assembly for synaptic vesicle clustering. $\mathrm{J}$ Biol Chem 2017, 292:14334-14348.

59. Xing G, Li M, Sun Y, Rui M, Zhuang Y, Lv H, Han J, Jia Z, Xie W: Neurexin-Neuroligin 1 regulates synaptic morphology and functions via the WAVE regulatory complex in Drosophila neuromuscular junction. Elife 2018, 7.

60. Banerjee S, Venkatesan A, Bhat MA: Neurexin, Neuroligin and Wishful Thinking coordinate synaptic cytoarchitecture and growth at neuromuscular junctions. Mol Cell Neurosci 2017, 78:9-24.

61. Marques G, Bao H, Haerry TE, Shimell MJ, Duchek P, Zhang B, O'Connor MB: The Drosophila BMP type II receptor Wishful Thinking regulates neuromuscular synapse morphology and function. Neuron 2002, 33:529-543.

62. Tapia-Rojas C, Inestrosa NC: Wnt signaling loss accelerates the appearance of neuropathological hallmarks of Alzheimer's disease in J20-APP transgenic and wild-type mice. J Neurochem 2018, 144:443-465.

63. Sindi IA, Tannenberg RK, Dodd PR: Role for the neurexin-neuroligin complex in Alzheimer's disease. Neurobiol Aging 2014, 35:746-756.

64. Martinez-Mir A, Gonzalez-Perez A, Gayan J, Antunez C, Marin J, Boada M, Lopez-Arrieta JM, Fernandez E, Ramirez-Lorca R, Saez ME, et al: Genetic study of neurexin and neuroligin genes in Alzheimer's disease. J Alzheimers Dis 2013, 35:403-412.

\section{Figures}




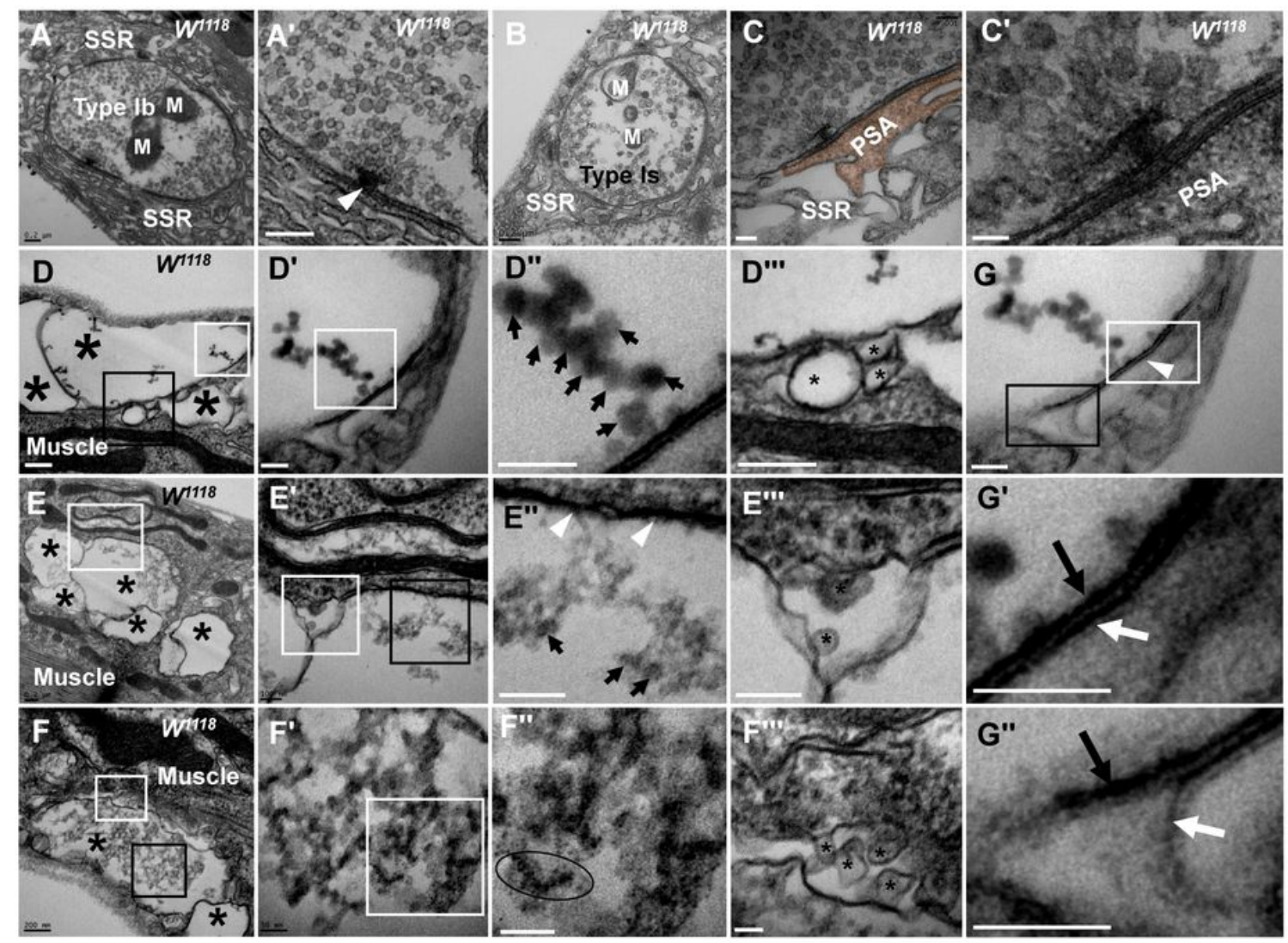

\section{Figure 1}

Thorough degeneration of NMJ boutonsin wild-typeDrosophila Normal type I boutons include type Ib boutons $(A)$ and type Is boutons $\left(A^{\prime}\right)$, with synapses and T-bars $\left(B\right.$, and $\left.C-C^{\prime}\right)$. Thoroughly degenerated NMJ boutons gather together and appear vacuolated, with extremely scarce SSR, in outer (D-D"', F-F"') and inner(E-E") muscle. Beaded dark synaptic vesicles overlap near a residual synapse (D"); agglomerated degenerated products with sparse dark synaptic vesicles ( $\left.E^{\prime \prime}\right)$; dark ultrafine particles of less than $2 \mathrm{~nm}$ in the degenerating bouton $\left(F^{\prime \prime}\right)$. The residual SSR membrane in degenerated boutons ( $\left.D^{\prime \prime}, E^{\prime \prime}, F^{\prime \prime \prime}\right)$.A residual synapse without T-bars (G-G") or a synaptic cleft.Large asteriskarrows show axon terminals, small asteriskarrows show residual SSR membrane, wedges show synapses or T-bars, black arrows show presynaptic membranes, and white arrows show postsynaptic membranes. Scale bars in A-B, D, E, F: 200 nm; C, D'-D"; E'-E"': 100 nm; C' F'-F", G-G": 50 nm. 

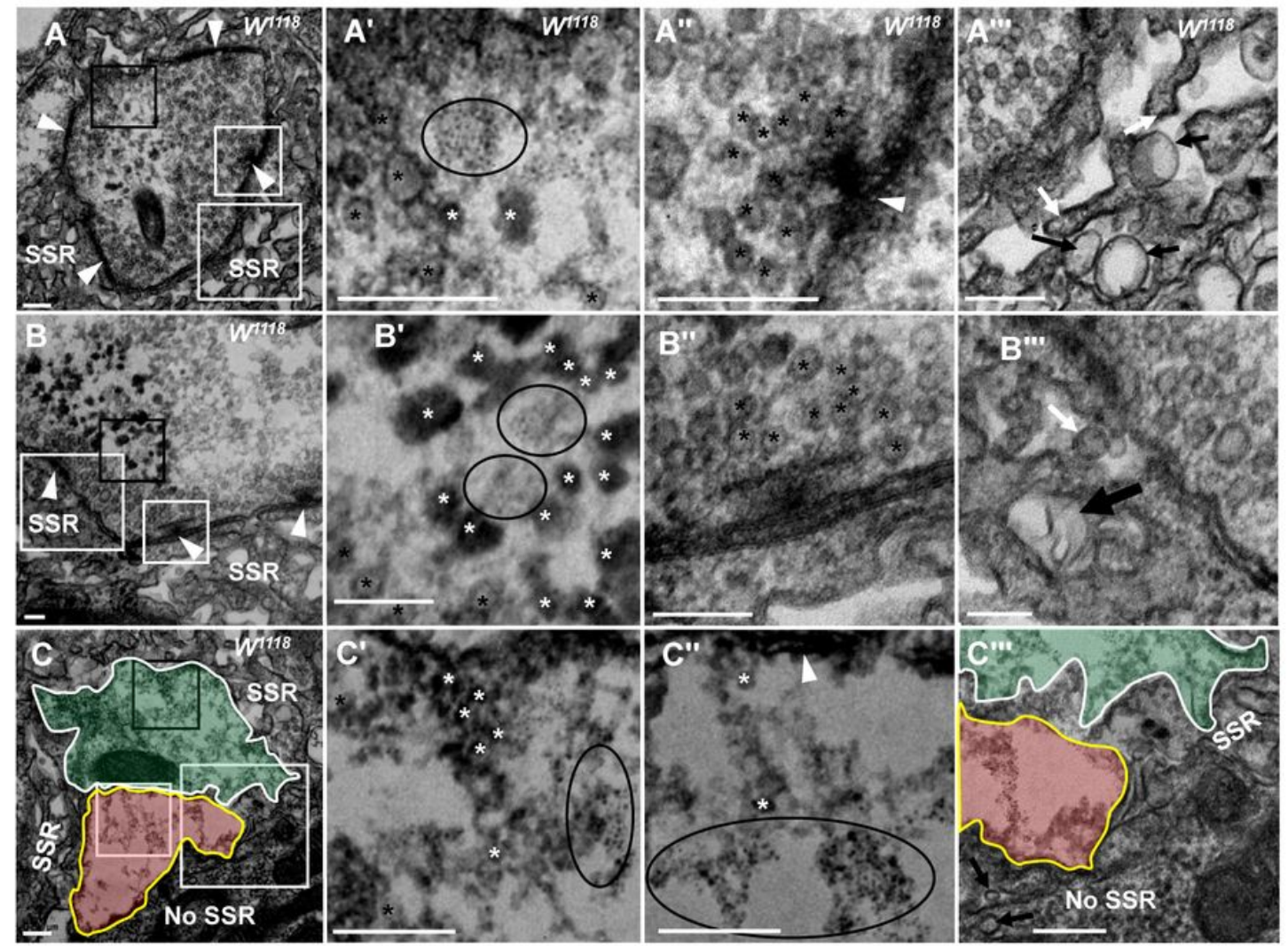

\section{Figure 2}

NMJ bouton is gradually degenerated in synaptic vesicles and SSR Fewer dark synaptic vesicles and dark ultrafine particles and more typical clear synaptic vesicles $\left(A, A^{\prime}\right)$ in a mildlydegenerated bouton with a typical T-bar (A, $\left.A^{\prime \prime}\right)$.Moredark synaptic vesicles and dark ultrafine particles and fewer typical clear synaptic vesicles $\left(B, B^{\prime}\right)$ in a moderatelydegenerated bouton with a typical T-bar (B, $\left.B^{\prime \prime}\right)$. Extremely rare clear synaptic vesicles, several dark synaptic vesicles, and many dark ultrafine particles (C, $\left.C^{\prime}, C^{\prime \prime}\right)$ in a severely degenerated bouton without T-bar structure (C).SSR membranes start withdrawing and swelling in a mildly degenerated bouton $\left(A^{\prime \prime}\right)$, fold inward in moderately degenerated bouton ( $\left.B^{\prime \prime \prime}\right)$, and become more disordered, looser or even missingin a severely degenerated bouton( $\left.\mathrm{C}^{\prime \prime \prime}\right)$. Black asteriskarrows show typical clear synaptic vesicles, white asteriskarrows show dark synaptic vesicles, and ellipses show dark ultrafine particles. White arrows show membrane withdrawal, thethin black arrow shows membrane swelling, and the thick black arrow shows membrane folding. Wedges show synapse or T-bar. The curves in panel $\mathrm{C}$ show two adjacent degenerated bouton, and the curve in panel $\mathrm{C}^{\prime \prime}$ shows a disordered and thin SSR membrane. $A^{\prime}, B^{\prime}, C^{\prime}$ arerespectively enlargement of the black box in $A, B, C . A^{\prime \prime}, B^{\prime \prime}, C^{\prime \prime}$ arerespectively enlargement of the small white box in $A, B, C . A^{\prime \prime \prime}, B^{\prime \prime \prime}, C^{\prime \prime \prime}$ arerespectively enlargement of the bigwhite box in

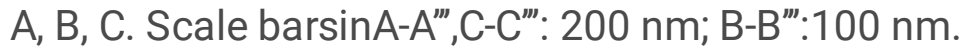




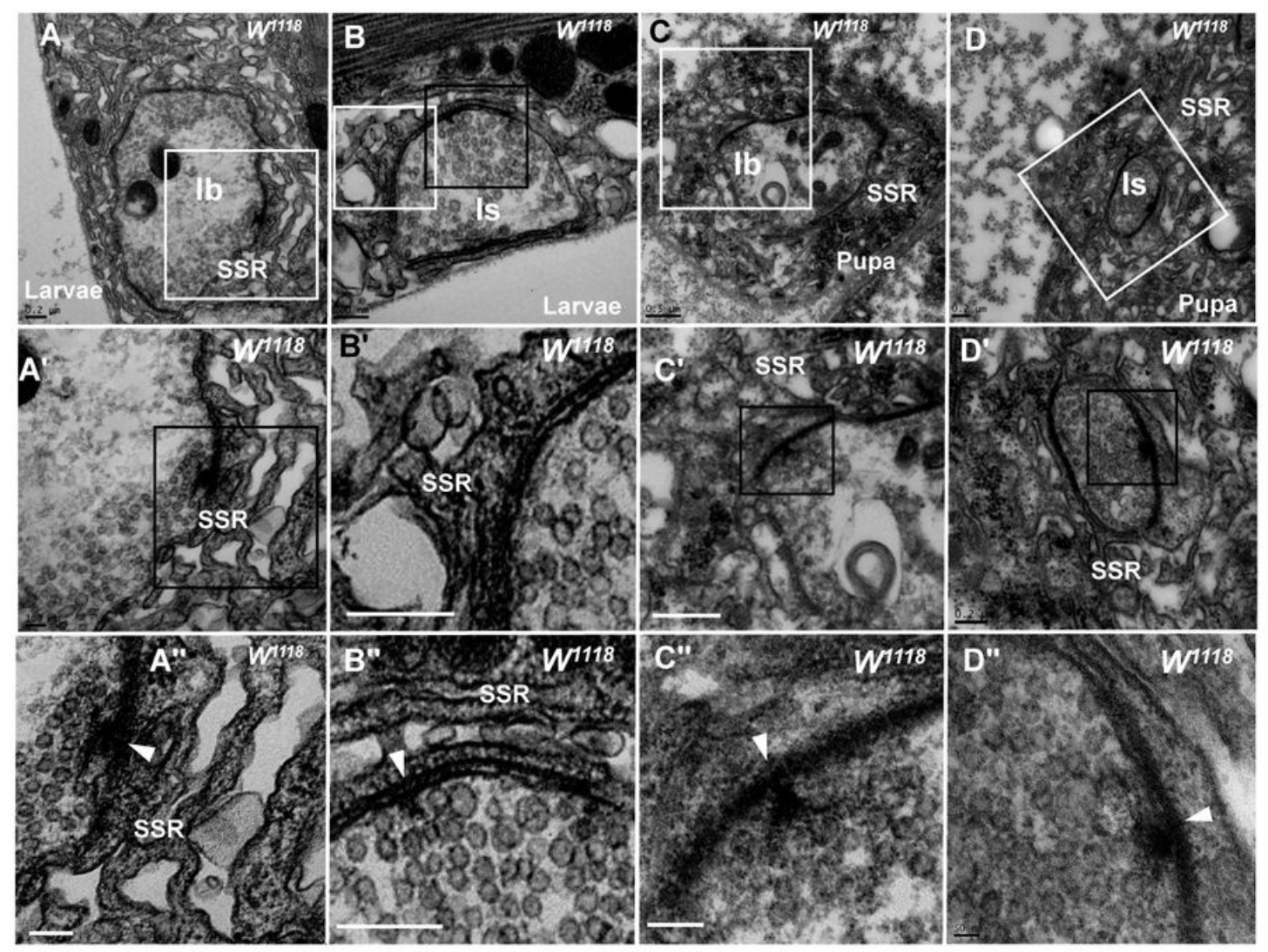

\section{Figure 3}

The initiation site of NMJ boutondegeneration There is a swollen and loose SSR membrane with typical clear vesicle and T-bar in a type lb bouton (A-A") and a type Is bouton (B-B") of the third-larval stage.There is an obviously swollen and loose SSR membrane with typical clear vesicles and T-bar in a type lb bouton (C-C") and a type Is bouton (D-D") in the pupa stage. Wedges show synapse or T-bar. A', B', C', D' arerespectively enlargement of the white box in $A, B, C, D$, and $A^{\prime \prime}, B^{\prime \prime}, C^{\prime \prime}, D^{\prime \prime}$ arerespectively enlargement of the black box in $A^{\prime}, B, C^{\prime}, D^{\prime}$. Scale bars inA, B-B", A" -B, D-D': 200 nm; A-A', C': 500 nm; D": 50nm. 


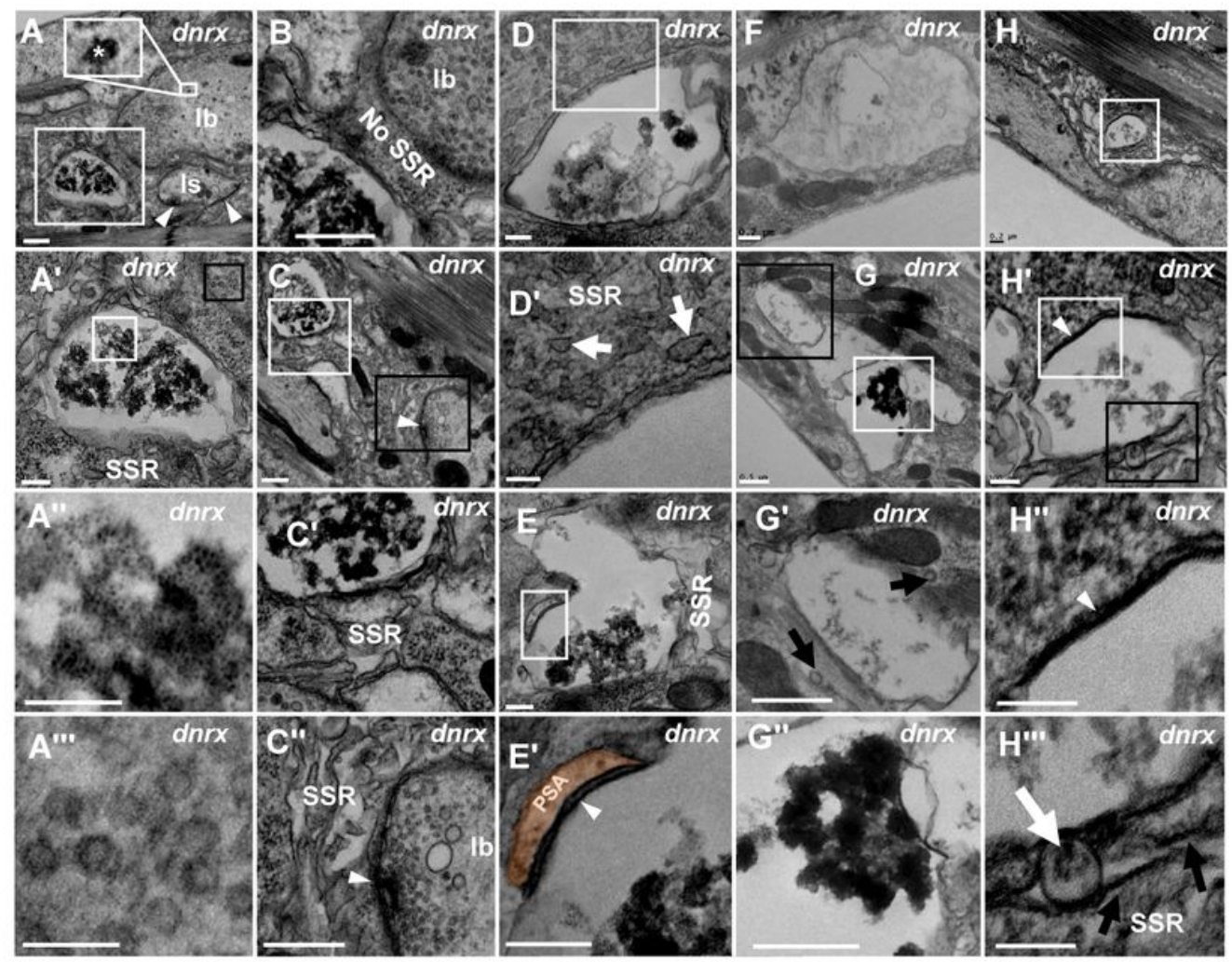

\section{Figure 4}

NMJ bouton is severelydegeneratedin thednrx mutant $A$ normal type $\mathrm{lb}$ bouton $(A)$ is adjacent to a degenerated bouton with obviously agglomerated dark ultrafine particles $\left(A^{\prime}, A^{\prime \prime}\right)$, filled with dense clear vesicles $\left(A^{\prime}, A^{\prime \prime \prime}\right)$. There is no SSR between thenormal bouton and the degenerated bouton (B). The retracted SSR membrane near the degenerated bouton becomebovious (C, $\left.C^{\prime}\right)$. The SSR membrane is relatively intact near the type lb bouton with a T-bar and clear vesicles $\left(C, C^{\prime \prime}\right)$. The seriously degenerated boutonhas swollen, discontinuous remnants of SSR membrane (D, D'). There are linear SSR remnants (E, $\left.E^{\prime}, E^{\prime \prime}\right)$ in a degenerated bouton. The irregular appearance of degenerated ghost boutons (F-H') $)$.Sparse (F, $\left.G^{\prime}\right)$ and agglomerated (G, G") dark ultrafine particles in axon terminal, andthin SSR membrane near the ghost bouton $\left(\mathrm{G}^{\prime}\right)$. The degenerationoccurs in a developing bouton in which there are dark ultrafine particles $\left(H, H^{\prime}\right)$, completely degenerated synapse $\left(H, H^{\prime \prime}\right)$, and retracted and linear SSR membrane $(H$, $\left.H^{\prime \prime \prime}\right)$.Wedges show synapse or T-bar. The curves in panel $E^{\prime}$ showshrinkingPSA. White arrows show swelling SSR membrane, and black arrows show retracted SSR membrane. A', A', C', D', E', G', $H^{\prime}$ and $H^{\prime \prime}$ are enlargement of the white box in $A, A^{\prime}, C, D, E, G, H$ and $H^{\prime}$. And $A^{\prime \prime}, G^{\prime \prime}$ and $H^{\prime}$ arerespectivelyenlargement of the black box in $A^{\prime}, G$ and $H^{\prime}$. Scale bars inA, B, C-C", G-G": 500 nm; A', D, E-E”, F, H: 200nm; A"-A"', D', H'-H'”: 100nm. 


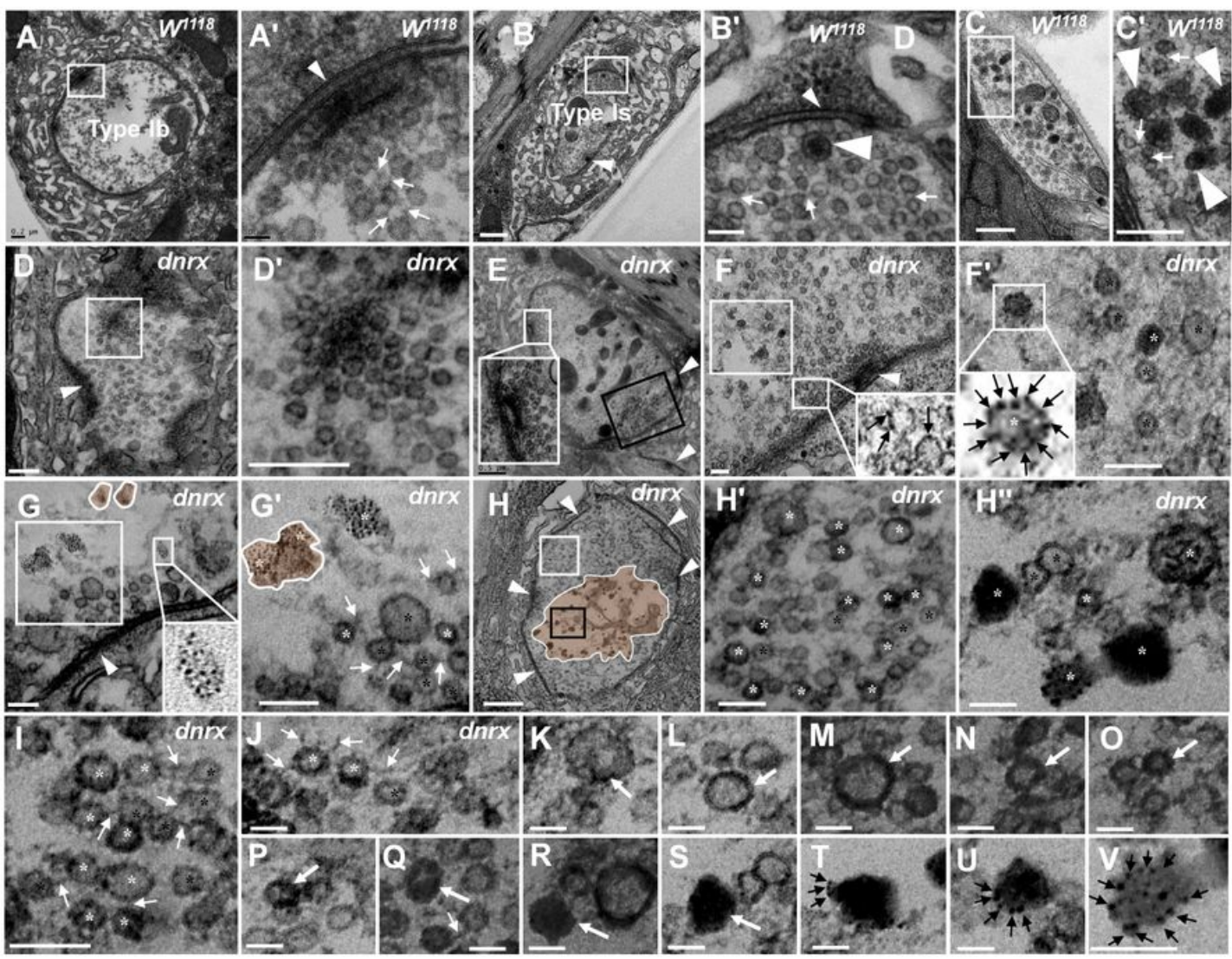

\section{Figure 5}

The processes ofNMJ boutondegeneration in dnrx The clear synaptic vesiclesand T-bar in type lb bouton $\left(A-A^{\prime}\right)$, dense core vesicles in type Is $\left(B-B^{\prime}\right)$ and type II bouton (C-C'), most clear synaptic vesicles were clustered with actin filament in wide-type $\left(A^{\prime}, B^{\prime}, C^{\prime}\right)$. T-bar (D) detaches from the presynaptic membrane and floatsin terminal with dense synaptic vesicles ( $\left.D^{\prime}\right)$. T-bar, be liked to detach, clusters dense synaptic vesicles before presynaptic membrane $(E)$, and some synaptic vesicles in the same bouton deviate from presynaptic membrane (E, black box). The first mode of synaptic vesicles degeneration ( $\left.F-G^{\prime}\right)$. The synaptic vesicles degenerate at the membranewithout actin filament $\left(F, F^{\prime}\right)$. And the ultra-fine spots dispersed into ellipse $(G)$ or irregular profile with dark ultrafine particles $\left(G^{\prime}\right)$. The second mode of synaptic vesicles degeneration $(\mathrm{H}-\mathrm{V})$. The degenerating type $\mathrm{lb}$ bouton without $\mathrm{T}-\mathrm{bar}(\mathrm{H})$ was full with clear vesicles and different dense vesicles $\left(\mathrm{H}^{\prime}\right)$ inthecortex of theaxon terminal $\left(\mathrm{H}-\mathrm{H}^{\prime}\right)$, and clear vesicles, dense vesicles, dark clumps without membraneand the dark ultrafine particles were distributedin the center of the terminal $\left(\mathrm{H}, \mathrm{H}^{\prime \prime}\right)$.Clear vesicles and different dense vesiclesare clustered actin filamentsnear the presynaptic membrane $(\mathrm{I})$, or detach each other without actin filaments $(\mathrm{J})$.A short dark linear occurs $(\mathrm{K})$ and expand $(\mathrm{L})$ to whole the membrane $(\mathrm{M}-\mathrm{N})$, the electron dense gradually expandinwardvesicles $(\mathrm{O}, \mathrm{P}$ $Q)$. The vesicle is full with electron densewithout membrane and actin filament $(R)$. The darker clump (S)separates several dark ultrafine particles at the edge of clump $(T)$. The ultrafine particles increase at the edge of clump $(U)$, until manydark ultrafine particlesshow up $(V)$. Small wedges show synapse or Tbar, large wedges show dense core vesicles, black arrows showultrafine particles, thin white arrowsshowfilaments, thick white arrowsshow the process of vesicle degeneration with the second mode 
degeneration, curves in panel G-G' show degenerated vesicle with the first mode of degeneration, and the curves in panel $\mathrm{H}$ shows the degeneration occurs in the center of terminal. Black asteriskarrows show typical clear synaptic vesicles, white asteriskarrows show different dense vesicles, dark clump or gathered dark ultrafine particles. $A^{\prime}-H^{\prime}$ arerespectivelyenlargement of the white box in $\mathrm{A}-\mathrm{H}, \mathrm{H}^{\prime \prime}$ is an enlargement of the black box in $\mathrm{H}$, and I-V are all enlarged from H. Scale bars inA, C-C', D-D': 200nm; B, H: 500nm; A', H'-H”, J-V: 50nm; B', F-F', G-G', I: 100nm.

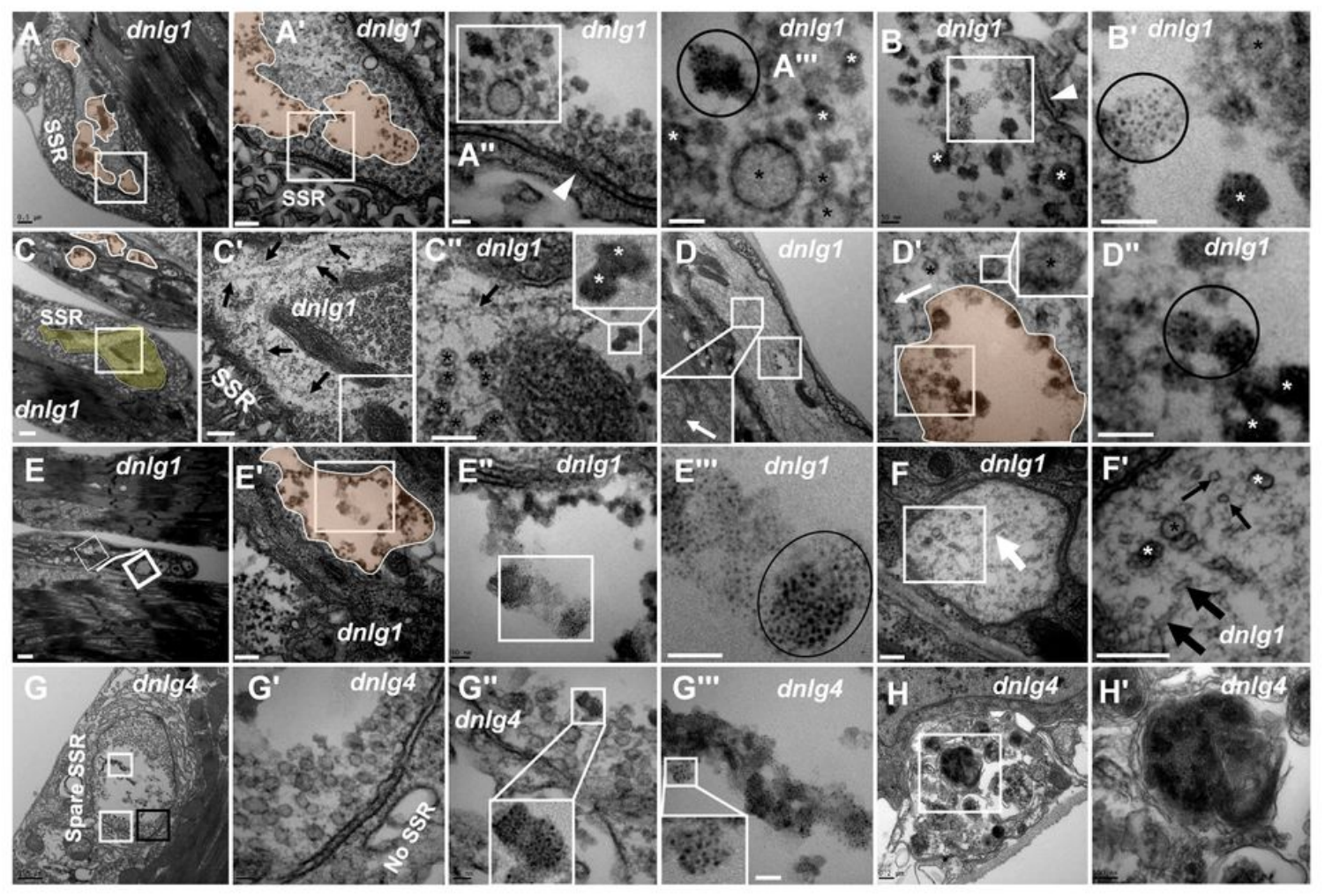

\section{Figure 6}

dnlg1and dnlg4 mutants lead to the degeneration of NMJ bouton The degeneratedaxon terminal has several plaques in degenerating type lb bouton with relatively intact SSR (A). Dark synaptic vesicles and dark ultrafine particles scatter inthe plaques of the degenerating axon terminal ( $\left.A^{\prime}-A^{\prime \prime}, B-B^{\prime}\right)$. Long microtubules protruded into a degenerating type $\mathrm{lb}$ bouton in above and below directions (C- $\left.\mathrm{C}^{\prime}\right)$, and the below microtubule passed through dense clear vesicles $\left(C^{\prime}-C^{\prime \prime}\right)$ with dark synaptic vesicle $\left(C^{\prime \prime}\right)$. A white plaques occurs in a large axon with different directions microtubules (D), contains dark synaptic vesicles and dark ultrafine particles ( $\left.D^{\prime}-D^{\prime \prime}\right)$, the clear vesicles are out of the white plaque $\left(D^{\prime}\right)$.Degenerated axons $(E)$ arefull with dark ultrafine particles $\left(6 E^{\prime}-E^{\prime \prime}\right)$, the others axon are intact with clear vesicles and different direction microtubules $\left(E, F-F^{\prime}\right)$. The degenerated type $\mathrm{lb}$ bouton is showed with retracted SSR,dark vesicles (G-G') and dark ultrafine particles (G, G"-G') in dnlg4. The autophagosomeis showed in a degenerated axon $\left(\mathrm{H}-\mathrm{H}^{\prime}\right)$.Wedges show synapse or T-bar, white arrow shows bent microtubule, thinblack arrows show microtubule transection,thick black arrows show microtubule non-transverse. The curves in panel $\mathrm{C}$ show degenerating terminal, ellipses show clumps of ultrafine particles. Black asteriskarrows 
show clear synaptic vesicles, white asteriskarrows show different dark synaptic vesicles. $A^{\prime}, A^{\prime \prime}, A^{\prime \prime \prime}, B^{\prime}, C^{\prime}$, $C^{\prime \prime}, D^{\prime}, D^{\prime \prime}, E^{\prime}, E^{\prime \prime}, E^{\prime \prime}, F^{\prime}, G^{\prime}, G^{\prime \prime}, G^{\prime \prime \prime}$ and $H^{\prime}$ arerespectivelyenlargement of the white box in $A, A^{\prime}, A^{\prime \prime}, B, C, C^{\prime}, C^{\prime \prime}, D$, $D^{\prime}, E, E^{\prime}, E^{\prime \prime}, F, G, G^{\prime}, G^{\prime \prime}$, and H. Scale bars inA, C, D, G: 500 nm; A', C', E', F-F', H: 200 nm; A"-A'”, B-B', D'-D”, E'E'”, G'-G'": 50nm; C", H': 100nm; E: 1000nm.

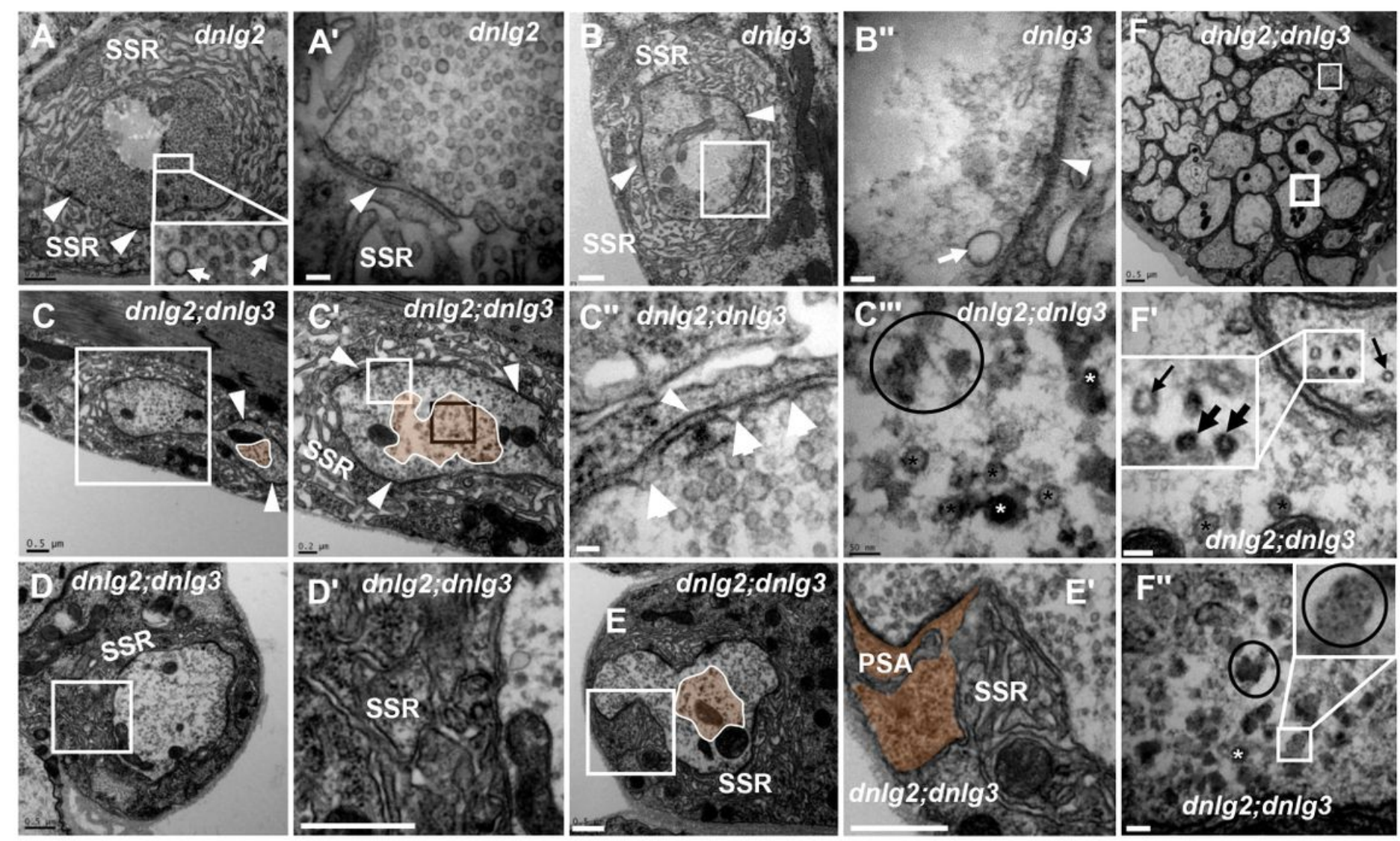

\section{Figure 7}

dnlg2and dnlg3 co-regulate degenerationin Drosophila NMJ There is no degeneration NMJ bouton in dnlg2 (A-A') and dnlg3 (B-B'). In dnlg2;dnlg3double mutant, the terminal has typical T-bar with rufflesof presynaptic membrane $\left(C-C^{\prime \prime}\right)$, and the dark vesicles in the center $\left(C-C^{\prime}, C^{\prime \prime \prime}\right)$, the SSR disorders (D-D'), retracts to form a large PSA (E-E'). The degeneration axons emerges in the outer of the fiber with dark vesicles and dark ultrafine particles $\left(F, F^{\prime \prime}\right)$. Inside of fiber, the periphery of axon is relatively intact with clear vesicles $\left(F-F^{\prime}\right)$, but most inside axonsare disordered without dark vesicles and dark ultrafine particles (F). Wedges show synapse or T-bar, white arrowsshow presynaptic ruffles, thinblack arrows showtransection of normal microtubule,thick black arrowstransection of degraded microtubule.The curves in panel $\mathrm{C}$ show degenerating terminal, ellipses show clumps of ultrafine particles. Black asteriskarrows show clear synaptic vesicles, white asteriskarrows show different dark synaptic vesicles. $A^{\prime}, B^{\prime}, C^{\prime}, C^{\prime \prime}, D^{\prime}$, and $E^{\prime}$ arerespectivelyenlargement of the white box in $A, B, C, C^{\prime}, D, E$. C'm is an enlargement of the black box in $C^{\prime} . F^{\prime}$ and $F^{\prime \prime}$ arerespectivelyenlargement of the thick white box and thin white box in $F$. Scale bars inA,B,C,D-D', E-E', F: 500 nm; A', B', C': 200 nm; C'-C'”, F'-F”: 50nm. 

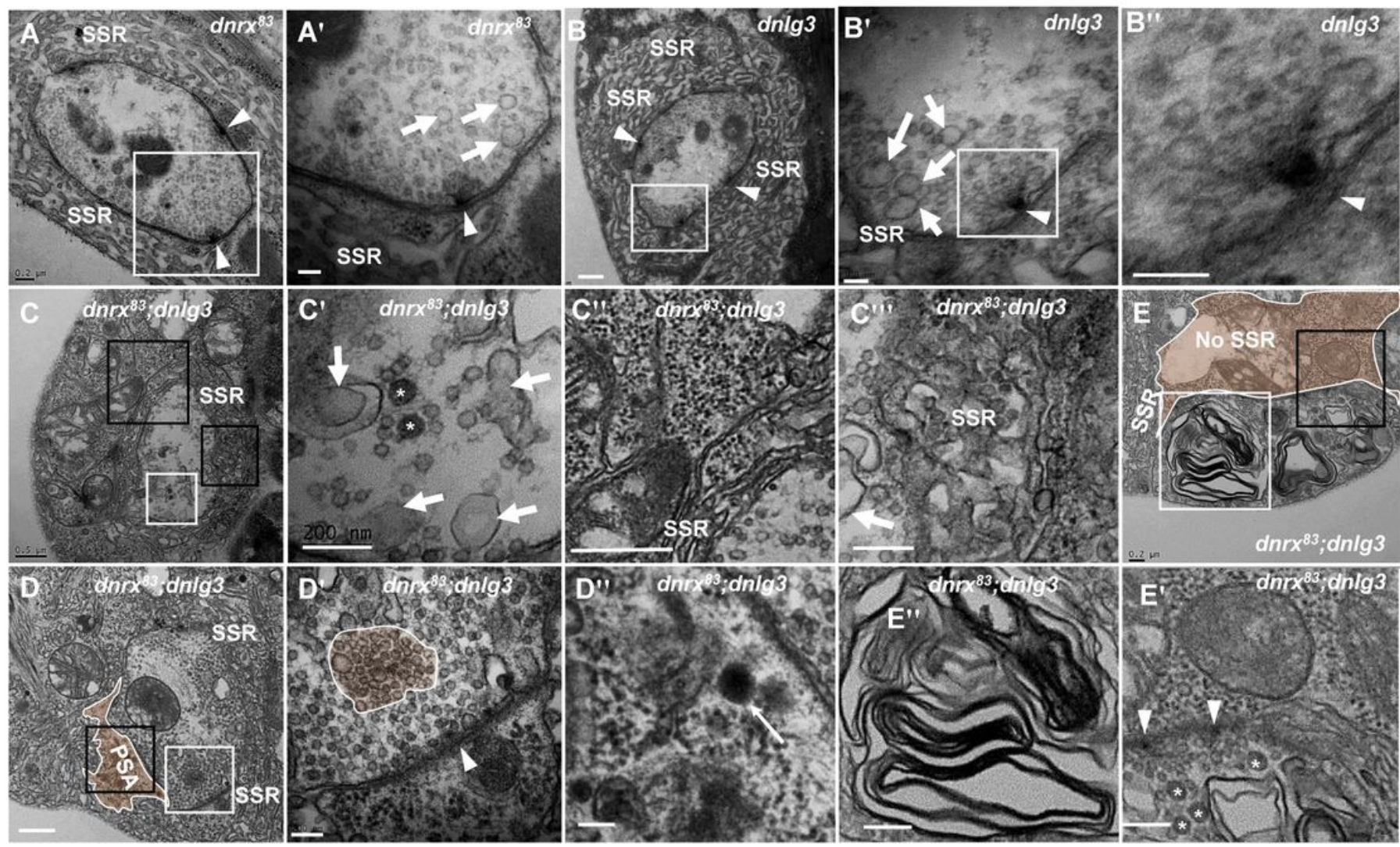

\section{Figure 8}

dnrxand dnlg3 co-lead degenerationin Drosophila NMJ There is no degeneration NMJ bouton in dnrx83(A-A') and dnlg3 (B-B"). Indnrx83;dnlg3double mutant, the terminal has dark vesicles and rare clear vesicles (C- $\left.\mathrm{C}^{\prime}\right)$, the SSR retracts to form rare SSR membrane and large PSA (C", D), and the dense SSR are disordered $\left(C^{\prime \prime}\right)$. T-bardetaches from presynaptic membrane (D- $\left.D^{\prime}\right)$, and lysosome is showed in SPA ( $D$, $\left.\mathrm{D}^{\prime \prime}\right)$.A degenerated type Is boutoncontains T-bar, dark vesicles(E-E'), and myelin-like mitochondria (E-E"), but barely SSR membrane. White arrows large clear vesicles, white asteriskarrows show dark synaptic vesicles. Curves in panel $D^{\prime}$ show detached T-bar with synaptic vesicles. A', B', B", C', D', and E' arerespectivelyenlargement of the white box in $A, B, B^{\prime}, C, D, E$. D" and E" arerespectivelyenlargement of the black box in $\mathrm{D}$ and $\mathrm{E}$. $\mathrm{C}^{\prime \prime}$ and $\mathrm{C}^{\prime \prime}$ arerespectivelyenlargement of the big black box and small black box in C. Scale bars inA, C', C", E-E": 200nm; A', B-B", D'-D":100nm; C, C", D: 500nm. 


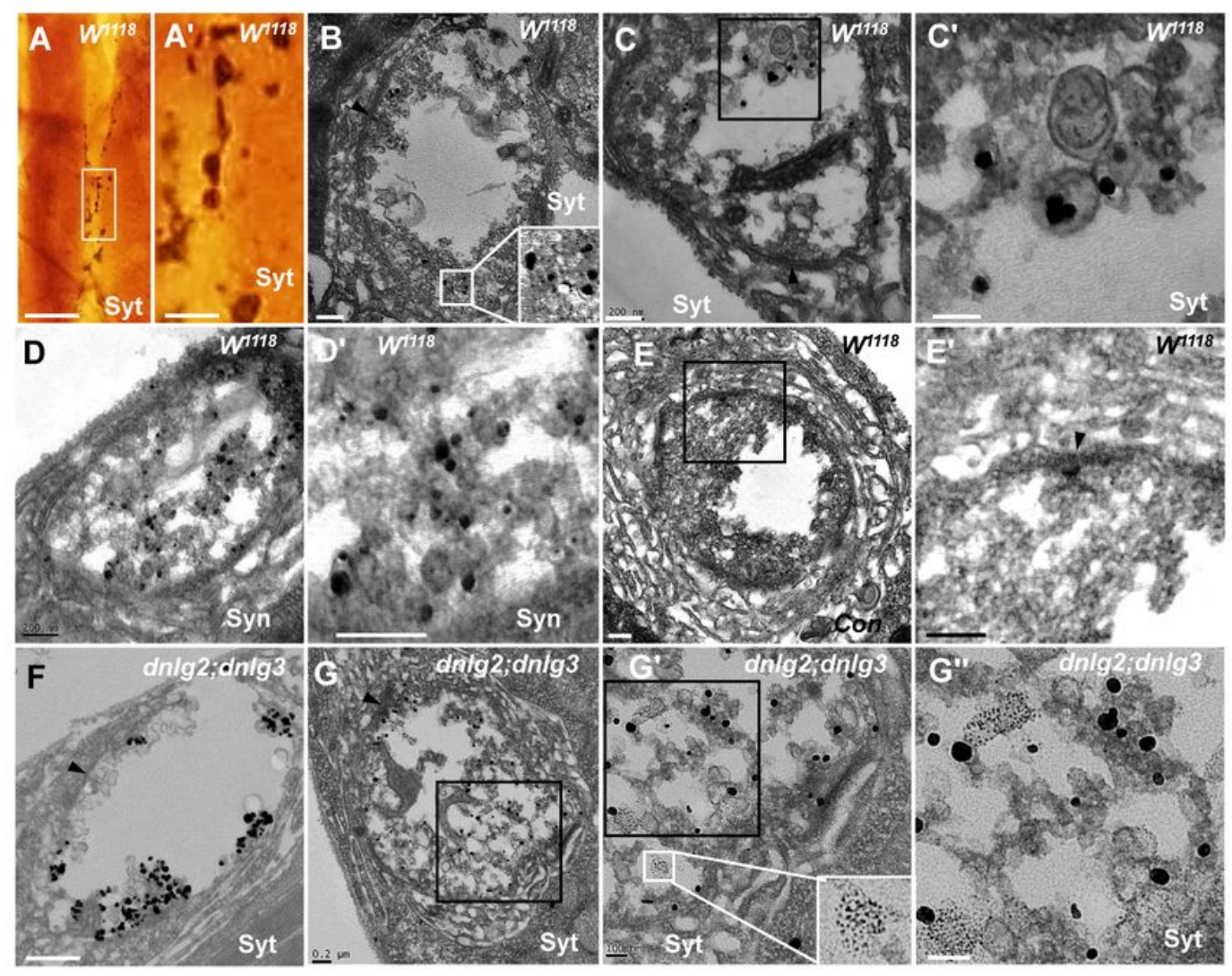

\section{Figure 9}

Synaptotagminare not distributed inultrafine particles in degenerated bouton Syt is present in the NMJ bouton in wild-type under light microscopy (A-A'), Syt(B-C') and Syn (D-D') are present in thesynaptic vesicles in TEM, E-E' is control with pre-immunoelectron microscopy.Syt is present in synaptic vesicles $(\mathrm{F})$, but not inultrafine particles of the degenerated NMJ bouton in dnlg2;dnlg3(G-G"). Wedges show synapse or T-bar. $A^{\prime}, C^{\prime}, D^{\prime}, E^{\prime}, G^{\prime}$, and $G^{\prime \prime}$ arerespectivelyenlargement of the box in $A, C, D, E, G$, and $G^{\prime}$.Scale bars inA: $100 \mu \mathrm{m} ; A^{\prime}: 20 \mu \mathrm{m} ; \mathrm{B}, \mathrm{C}, \mathrm{D}-\mathrm{E}^{\prime}, \mathrm{G}: 200 \mathrm{~nm} ; \mathrm{C}^{\prime}, \mathrm{G}^{\prime}-\mathrm{G}^{\prime \prime}:$ 100nm; F: 500nm. 


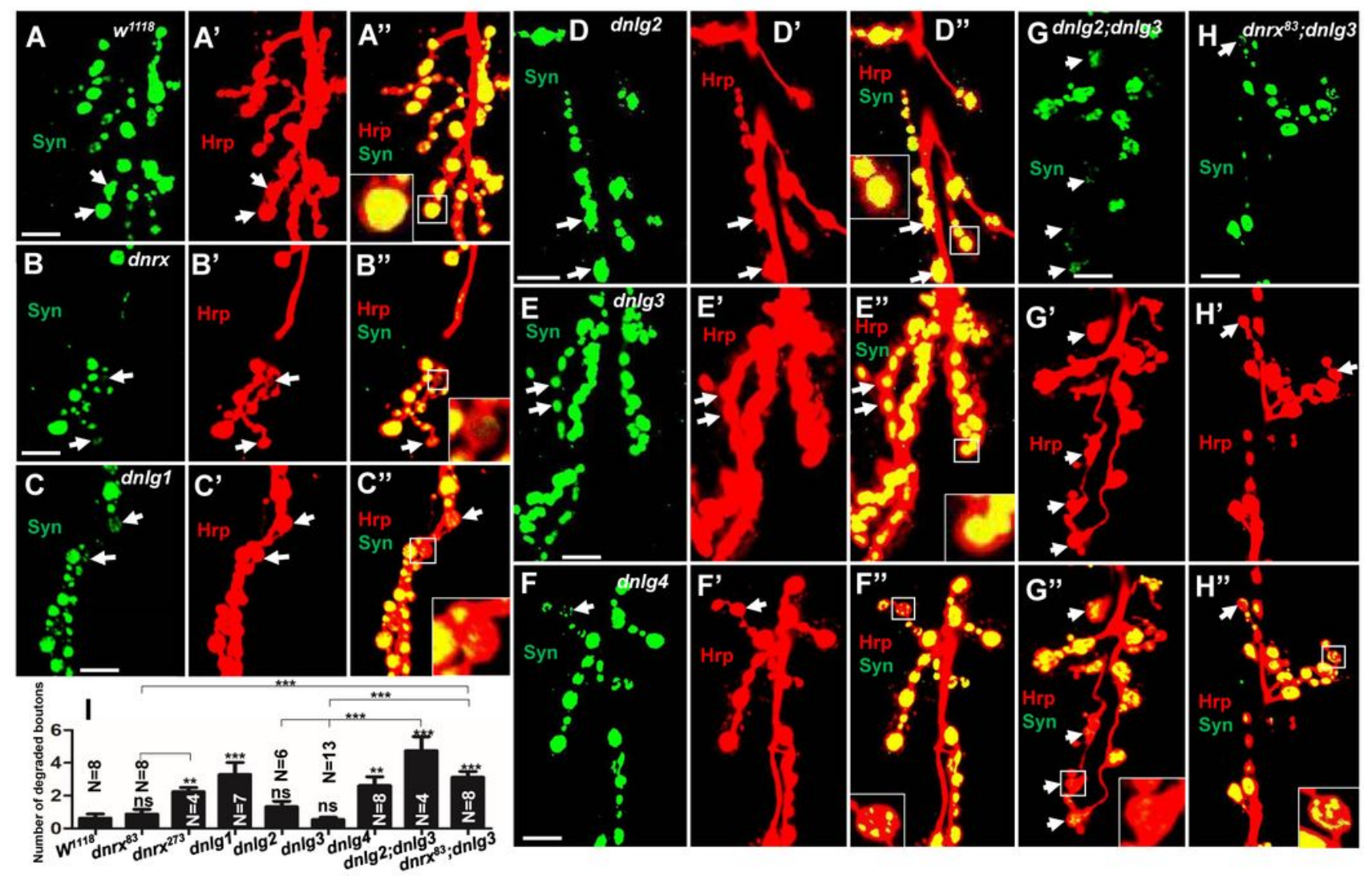

Figure 10

Neurexin and Neuroliginsjointly regulate type lb bouton degeneration Almost no degeneration in type lb bouton in wild-type (A-A") and dnlg2 (D-D") and dnlg3(E-E"). The degenerated type lb bouton in dnrx273(B$\left.B^{\prime \prime}\right)$, dnlg1(C-C"), dnlg4(F"), dnlg2;dnlg3(G-G") and dnrx83;dnlg3(F-F"). Quantity statistics of degenerated type $\mathrm{lb}$ bouton $(\mathrm{I})$. Thewhite arrows show the degenerated type $\mathrm{lb}$ bouton. Scale bars, $10 \mu \mathrm{m}$. ${ }^{\star \star}, \mathrm{P}<0.01$; $\star \star \star, P<0.001 ; n s$, no significance. 

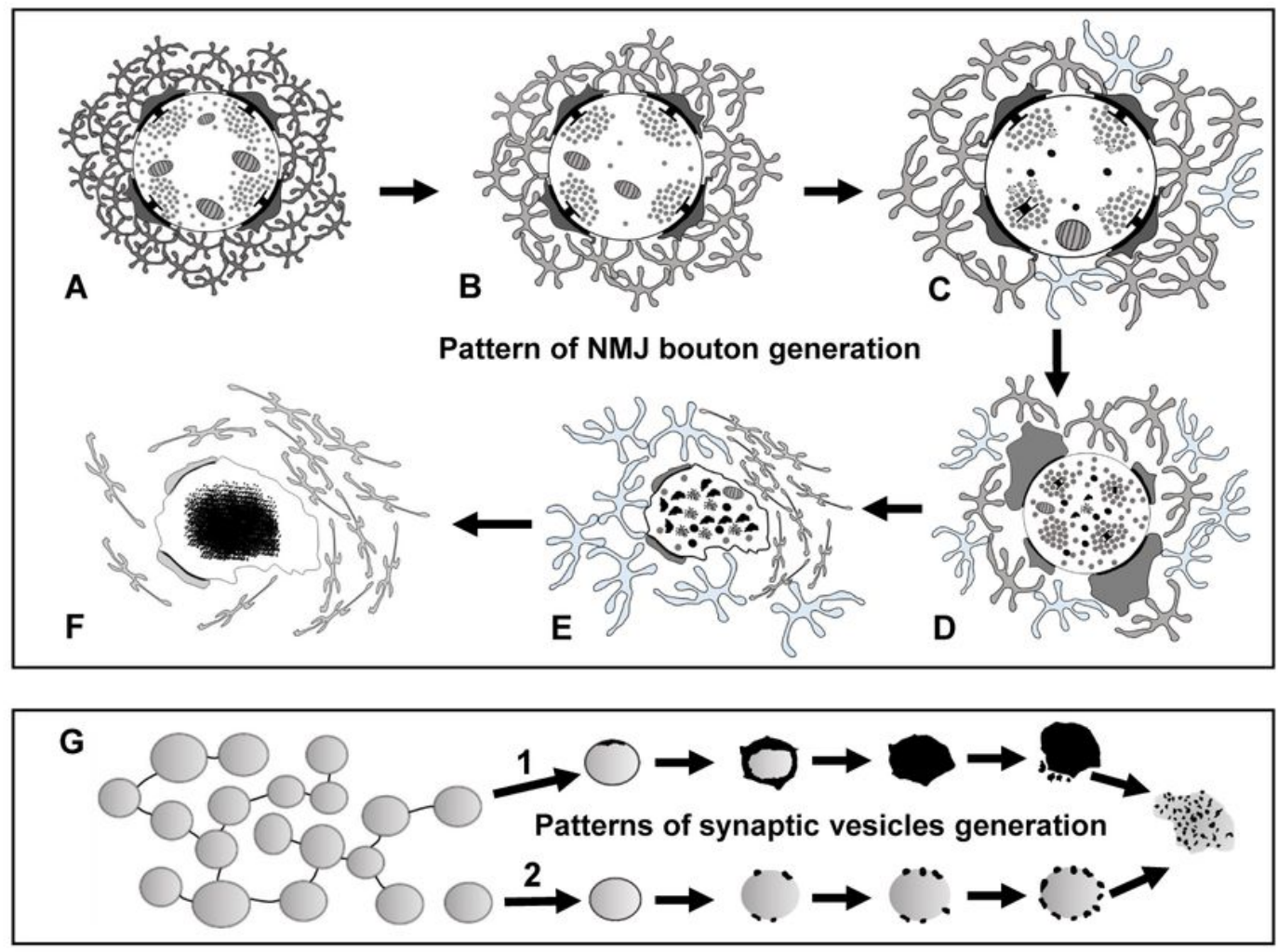

औ้ Regular dense SSR

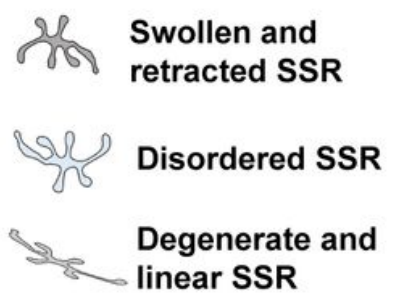

Synapse with T-bar, PSA, clear vesicles

Degenerate synapse with PSA

dark Vesicle or clump

$\therefore \quad$ Vesicle with dark spots

第 Dark ultrafine particles

(1D) Mitochondria

\section{Figure 11}

The degeneration model for Drosophila NMJ synapse Type lb bouton contains T-bars, dense clear synaptic vesicles, mitochondria, and regular SSR with PSA (A).The SSR membranes start to swell and retract, along with reduced mitochondria and synaptic vesicles(B). The T-bar detacheswith the swelling mitochondria, and degenerating vesicles appear in the center of terminal and SSR get further swelling and retraction (C). All the T-barsdetach with cluster the clear synaptic vesicles, more dark vesiclesdeform and collapse into irregular dark vesicles without membrane and then fragment into dark ultrafine particles, and SSR more retracted and fluffy with the PSA expansion or reduction (D). The terminal deforms with more collapsed dark vesicles and residualsynaptic vesicles, mitochondria and PSA, and most SSR become loose and disordered, some of which degenerate into linear (E). All residualmembranesbecome very thin or degenerateinto linear, and all the residual elements in terminal degenerate in situ and eventually form a cluster of ultrafine particles(F). There are twomodes of synaptic vesicles degeneration $(G)$. First, the synaptic vesicles without actin filament degenerated on the membrane with ultra-fine spots, and collapsed and dispersed irregular profile with dark ultrafine particles.Second, the clear synaptic vesicle with actin filament degenerated into dense synaptic vesicle, formed irregular dark clump without membrane, and collapsed and dispersed irregular profile with dark ultrafine particles. The degeneration of NMJ bouton occurs in normal physiological conditions, but it wouldbe acceleratedindnrx and dnlgs. Furthermore, there is a synergistic effect indnrx;dnlgs double mutation and dnlg2; dnlg3 double mutant to promote degeneration of NMJ bouton.

\section{Supplementary Files}


This is a list of supplementary files associated with this preprint. Click to download.

- Supplementarydata.docx

- FigureS1.tif

- Figures4.tif

- FigureS3.tif

- Figures2.tif 